\title{
Organotellurium(VI) azides and halides
}

\author{
Thomas M. Klapötke, * Burkhard Krumm, Kurt Polborn, and Ingo Schwab
}

\section{Supporting Information}

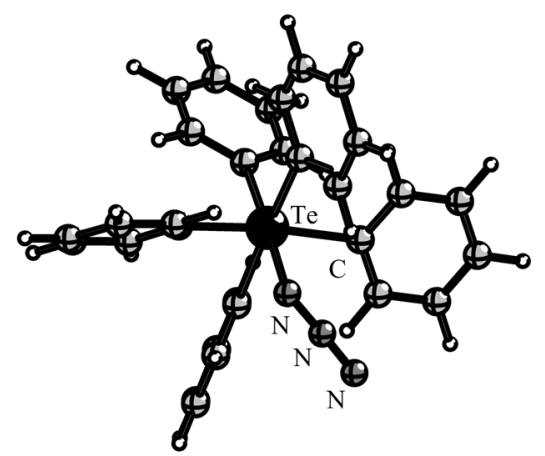

(1)

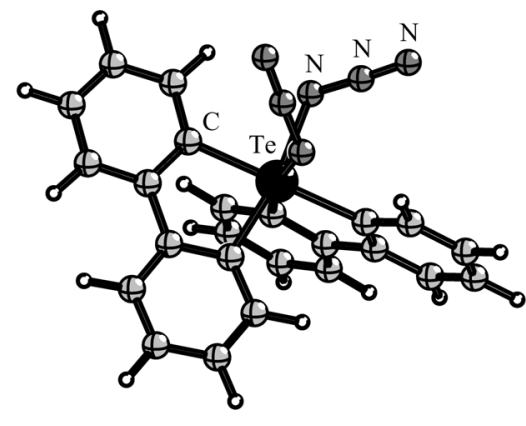

(2)

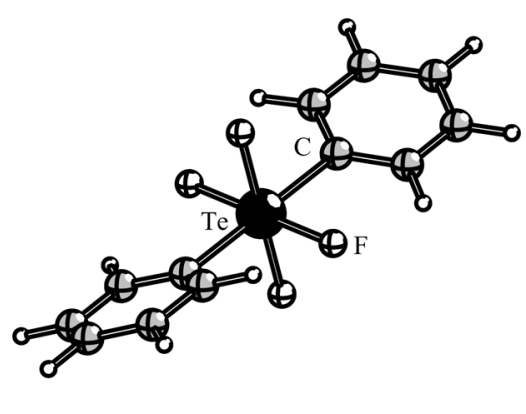

(3)

Calculated structures of $\mathrm{Ph}_{5} \mathrm{TeN}_{3}\left(\mathbf{1}, C_{1}\right)$, biphen $2 \mathrm{Te}\left(\mathrm{N}_{3}\right)_{2}\left(\mathbf{2}, C_{1}\right)$ and trans $-\mathrm{Ph}_{2} \mathrm{TeF}_{4}\left(\mathbf{3}, D_{2}\right)$ at the B3LYP/cc-VDZ level.

Cartesian coordinates of the B3LYP/cc-pVDZ $(\mathrm{PP}(\mathrm{Te})=\mathrm{MDF} 28)$ minimum structures for $\mathrm{Ph}_{5} \mathrm{TeN}_{3}$ $\left(\mathbf{1}, C_{1}\right)$, biphen $2 \mathrm{Te}\left(\mathrm{N}_{3}\right)_{2}\left(\mathbf{2}, C_{1}\right)$ and trans $-\mathrm{Ph}_{2} \mathrm{TeF}_{4}\left(\mathbf{3}, D_{2}\right)$ :

$\begin{array}{lrrr}59 & & & \\ \text { ph5ten }-\mathrm{b} 2 \mathrm{f} & & \\ \mathrm{Te} & 0.015028 & -0.030696 & -0.179852 \\ \mathrm{C} & 0.144349 & -0.272037 & 2.023219 \\ \mathrm{C} & 0.151618 & -1.546702 & 2.609528 \\ \mathrm{C} & 0.223265 & 0.857115 & 2.851735 \\ \mathrm{C} & 0.235469 & -1.687037 & 3.999859 \\ \mathrm{H} & 0.098006 & -2.443024 & 1.991313 \\ \mathrm{C} & 0.302122 & 0.712944 & 4.241986 \\ \mathrm{H} & 0.225059 & 1.859969 & 2.423801 \\ \mathrm{C} & 0.308964 & -0.558597 & 4.820103 \\ \mathrm{H} & 0.243242 & -2.687628 & 4.439099 \\ \mathrm{H} & 0.359884 & 1.603871 & 4.872128 \\ \mathrm{H} & 0.372295 & -0.669796 & 5.905024 \\ \mathrm{C} & -2.211217 & 0.284915 & -0.125752 \\ \mathrm{C} & -2.837651 & 0.814637 & 1.008120\end{array}$




\begin{tabular}{|c|c|c|c|}
\hline C & -2.978841 & -0.081773 & -1.237168 \\
\hline C & -4.228341 & 0.980160 & 1.026998 \\
\hline $\mathrm{H}$ & -2.257002 & 1.101986 & 1.885887 \\
\hline C & -4.368084 & 0.086880 & -1.214726 \\
\hline $\mathrm{H}$ & -2.497968 & -0.508613 & -2.119004 \\
\hline $\mathrm{C}$ & -4.994807 & 0.618361 & -0.083985 \\
\hline $\mathrm{H}$ & -4.710079 & 1.393436 & 1.916745 \\
\hline $\mathrm{H}$ & -4.959779 & -0.200043 & -2.087512 \\
\hline $\mathrm{H}$ & -6.079345 & 0.749550 & -0.068290 \\
\hline $\mathrm{C}$ & 0.360719 & 2.182124 & -0.104789 \\
\hline $\mathrm{C}$ & -0.655418 & 3.069699 & -0.476129 \\
\hline $\mathrm{C}$ & 1.615778 & 2.680388 & 0.270264 \\
\hline C & -0.417940 & 4.450484 & -0.463642 \\
\hline $\mathrm{H}$ & -1.634926 & 2.702002 & -0.779652 \\
\hline $\mathrm{C}$ & 1.843900 & 4.060412 & 0.292264 \\
\hline $\mathrm{H}$ & 2.420698 & 2.001984 & 0.556823 \\
\hline C & 0.827794 & 4.947577 & -0.076562 \\
\hline $\mathrm{H}$ & -1.214473 & 5.134302 & -0.766022 \\
\hline $\mathrm{H}$ & 2.822039 & 4.440180 & 0.597908 \\
\hline $\mathrm{H}$ & 1.009766 & 6.024786 & -0.066082 \\
\hline C & 2.225103 & -0.354715 & -0.464413 \\
\hline C & 2.857790 & 0.083631 & -1.633738 \\
\hline C & 2.974108 & -0.991418 & 0.531821 \\
\hline C & 4.233264 & -0.113196 & -1.801867 \\
\hline $\mathrm{H}$ & 2.277142 & 0.568176 & -2.418383 \\
\hline C & 4.350532 & -1.187688 & 0.358480 \\
\hline $\mathrm{H}$ & 2.502581 & -1.337197 & 1.452456 \\
\hline $\mathrm{C}$ & 4.982381 & -0.749211 & -0.807509 \\
\hline $\mathrm{H}$ & 4.718015 & 0.231750 & -2.718698 \\
\hline $\mathrm{H}$ & 4.926371 & -1.684959 & 1.143138 \\
\hline $\mathrm{H}$ & 6.055805 & -0.902704 & -0.941567 \\
\hline C & -0.352474 & -2.196845 & -0.582213 \\
\hline $\mathrm{C}$ & 0.523399 & -2.899996 & -1.414048 \\
\hline $\mathrm{C}$ & -1.470143 & -2.841342 & -0.040352 \\
\hline C & 0.287558 & -4.251379 & -1.692431 \\
\hline $\mathrm{H}$ & 1.385868 & -2.401167 & -1.856917 \\
\hline $\mathrm{C}$ & -1.697181 & -4.196007 & -0.313068 \\
\hline $\mathrm{H}$ & -2.172208 & -2.298809 & 0.595472 \\
\hline C & -0.818996 & -4.902075 & -1.139762 \\
\hline $\mathrm{H}$ & 0.972382 & -4.793677 & -2.349022 \\
\hline $\mathrm{H}$ & -2.567522 & -4.695401 & 0.119975 \\
\hline $\mathrm{H}$ & -1.000071 & -5.957350 & -1.357387 \\
\hline $\mathrm{N}$ & -0.080710 & 0.093408 & -2.453444 \\
\hline $\mathrm{N}$ & -0.511205 & 1.095973 & -2.998754 \\
\hline $\mathrm{N}$ & -0.919424 & 2.006150 & -3.575268 \\
\hline
\end{tabular}

47

biphen 2 ten 6

$\begin{array}{lrrr}\text { C } & 0.074191 & 0.191515 & 0.069590 \\ \mathrm{C} & 0.064992 & 0.023014 & 1.466526 \\ \mathrm{C} & 1.211166 & 0.139539 & 2.247759 \\ \mathrm{C} & 2.429192 & 0.420405 & 1.615550 \\ \mathrm{C} & 2.470756 & 0.581482 & 0.227145 \\ \mathrm{C} & 1.309538 & 0.470119 & -0.540853 \\ \mathrm{C} & -1.213753 & 0.068032 & -0.666708 \\ \mathrm{C} & -2.374285 & -0.201576 & 0.080769 \\ \mathrm{C} & -3.624103 & -0.326140 & -0.520056 \\ \mathrm{C} & -3.732543 & -0.186513 & -1.908547 \\ \mathrm{C} & -2.589914 & 0.077495 & -2.670690 \\ \mathrm{C} & -1.340721 & 0.204213 & -2.059585 \\ \mathrm{H} & 1.147988 & 0.016915 & 3.329064\end{array}$




$\begin{array}{lrrr}\mathrm{H} & 3.339817 & 0.520725 & 2.209833 \\ \mathrm{H} & 3.419697 & 0.803005 & -0.266253 \\ \mathrm{H} & 1.370448 & 0.604611 & -1.621960 \\ \mathrm{H} & -4.511657 & -0.528000 & 0.083295 \\ \mathrm{H} & -4.707133 & -0.280769 & -2.392450 \\ \mathrm{H} & -2.671678 & 0.188414 & -3.754142 \\ \mathrm{H} & -0.465100 & 0.411829 & -2.676718 \\ \mathrm{Te} & -1.939927 & -0.395372 & 2.189849 \\ \mathrm{C} & -1.845937 & -2.555013 & 2.169495 \\ \mathrm{C} & -3.060981 & -3.132216 & 2.580308 \\ \mathrm{C} & -0.751097 & -3.330670 & 1.797754 \\ \mathrm{C} & -3.153305 & -4.534295 & 2.601464 \\ \mathrm{C} & -4.170169 & -2.217200 & 2.965974 \\ \mathrm{C} & -0.860292 & -4.725816 & 1.825648 \\ \mathrm{H} & 0.184078 & -2.858220 & 1.489872 \\ \mathrm{C} & -2.061199 & -5.319852 & 2.227038 \\ \mathrm{H} & -4.078344 & -5.022607 & 2.912141 \\ \mathrm{C} & -3.949447 & -0.829557 & 2.890716 \\ \mathrm{C} & -5.433492 & -2.649078 & 3.406270 \\ \mathrm{H} & -0.008787 & -5.346147 & 1.537559 \\ \mathrm{H} & -2.148896 & -6.408235 & 2.251260 \\ \mathrm{C} & -4.914233 & 0.107912 & 3.248441 \\ \mathrm{C} & -6.415917 & -1.721742 & 3.760563 \\ \mathrm{H} & -5.656033 & -3.714917 & 3.477382 \\ \mathrm{C} & -6.164051 & -0.348260 & 3.686650 \\ \mathrm{H} & -4.687731 & 1.172489 & 3.188460 \\ \mathrm{H} & -7.389376 & -2.078185 & 4.104550 \\ \mathrm{H} & -6.933855 & 0.369903 & 3.976690 \\ \mathrm{~N} & -2.401960 & 1.767500 & 2.434286 \\ \mathrm{~N} & -1.110740 & -0.426216 & 4.254390 \\ \mathrm{~N} & -1.480662 & 2.476328 & 2.820804 \\ \mathrm{~N} & -1.831177 & 0.031946 & 5.132883 \\ \mathrm{~N} & -0.654315 & 3.191459 & 3.173350 \\ \mathrm{~N} & -2.463786 & 0.441295 & 5.999372 \\ & & & \end{array}$

$\begin{array}{lrrr}27 & & & \\ \text { trans-ph2tef4, D2 } & \text { symmetry } & \\ \text { Te } & -0.000000 & 0.000000 & -0.000000 \\ \mathrm{C} & -0.000000 & -0.000000 & 2.132318 \\ \mathrm{C} & 1.225507 & 0.011953 & 2.798847 \\ \mathrm{C} & -1.225507 & -0.011953 & 2.798847 \\ \mathrm{C} & 1.212173 & 0.014015 & 4.197566 \\ \mathrm{H} & 2.161909 & 0.014642 & 2.243373 \\ \mathrm{C} & -1.212173 & -0.014015 & 4.197566 \\ \mathrm{H} & -2.161909 & -0.014642 & 2.243373 \\ \mathrm{C} & 0.000000 & -0.000000 & 4.893546 \\ \mathrm{H} & 2.159354 & 0.024530 & 4.741154 \\ \mathrm{H} & -2.159354 & -0.024530 & 4.741154 \\ \mathrm{H} & 0.000000 & -0.000000 & 5.985743 \\ \mathrm{C} & -0.000000 & 0.000000 & -2.132318 \\ \mathrm{C} & -1.095952 & 0.548544 & -2.798847 \\ \mathrm{C} & 1.095952 & -0.548544 & -2.798847 \\ \mathrm{C} & -1.083065 & 0.544546 & -4.197566 \\ \mathrm{H} & -1.936188 & 0.961893 & -2.243373 \\ \mathrm{C} & 1.083065 & -0.544546 & -4.197566 \\ \mathrm{H} & 1.936188 & -0.961893 & -2.243373 \\ \mathrm{C} & -0.000000 & 0.000000 & -4.893546 \\ \mathrm{H} & -1.929554 & 0.969657 & -4.741154 \\ \mathrm{H} & 1.929554 & -0.969657 & -4.741154 \\ \mathrm{H} & -0.000000 & 0.000000 & -5.985743 \\ \mathrm{~F} & 1.911652 & -0.441867 & -0.000000\end{array}$




$\begin{array}{rrrr}F & 0.439453 & 1.901210 & 0.000000 \\ F & -1.911652 & 0.441867 & 0.000000 \\ F & -0.439453 & -1.901210 & -0.000000\end{array}$




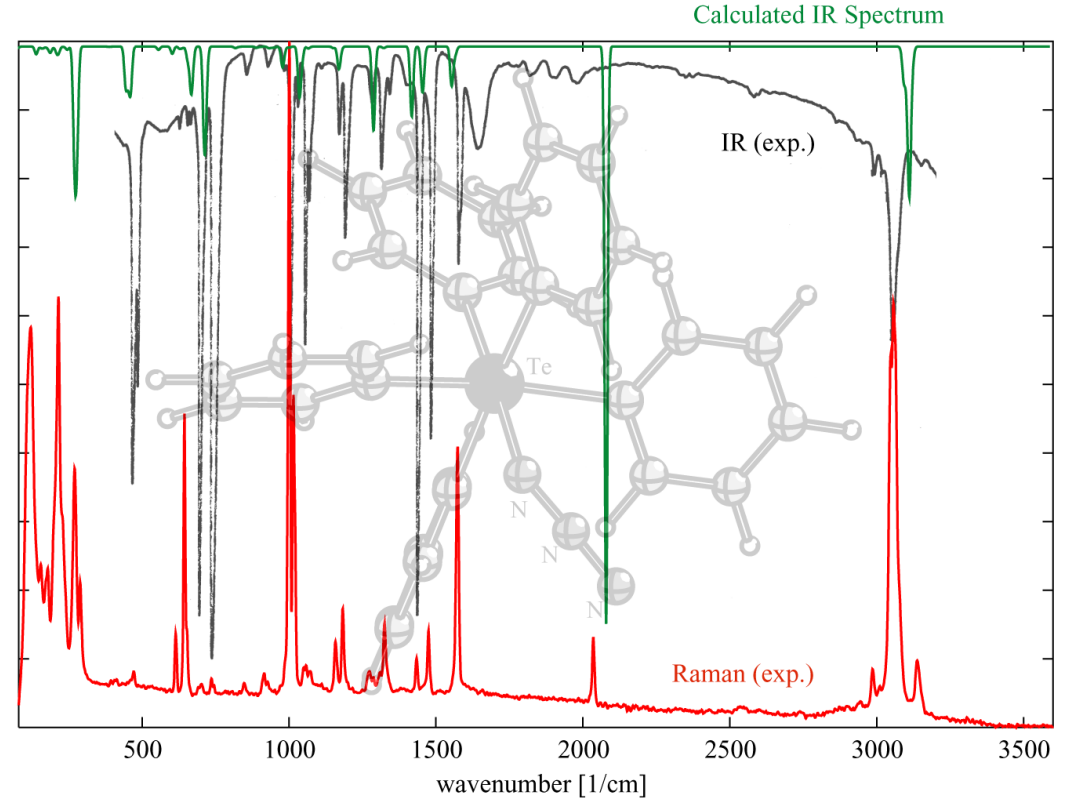

Calculated and experimental IR and Raman spectra of $\mathrm{Ph}_{5} \mathrm{TeN}_{3}(\mathbf{1})$, unscaled numerical frequency analysis with the SNF program at the RI-BP86/TZVP level of theory.

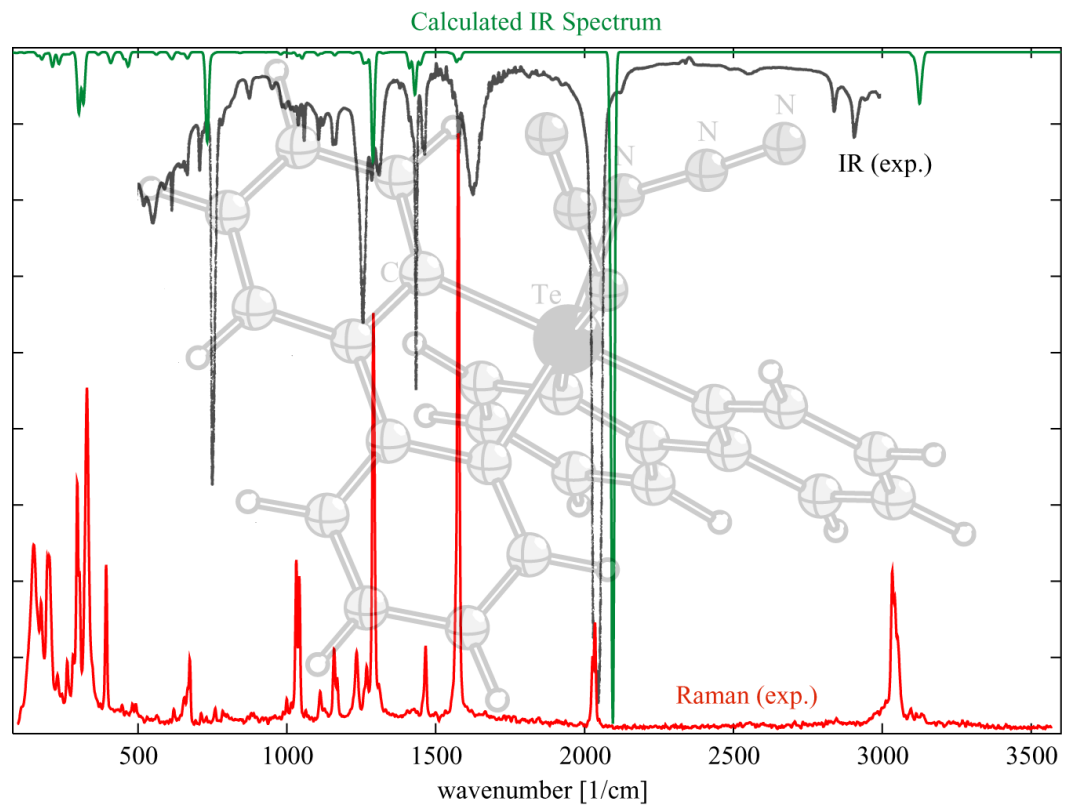

Calculated and experimental IR and Raman spectra of biphen ${ }_{2} \mathrm{Te}\left(\mathrm{N}_{3}\right)_{2}(2)$, unscaled numerical frequency analysis with the SNF program at the RI-BP86/TZVP level of theory. 
Analytical frequency analysis for trans $-\mathrm{Ph}_{2} \mathrm{TeF}_{4}\left(D_{2}\right)$ at the RI-MP2/TZVP level:

\begin{tabular}{|c|c|c|c|c|c|}
\hline mode & symmetry & wave number & IR intensity & select & rules \\
\hline & & $\mathrm{cm} * \star(-1)$ & $\mathrm{km} / \mathrm{mol}$ & IR & RAMAN \\
\hline 7 & b3 & 75.32 & 1.43310 & YES & YES \\
\hline 8 & $\mathrm{~b} 2$ & 75.39 & 1.43323 & YES & YES \\
\hline 9 & $\mathrm{~b} 1$ & 101.10 & 0.00001 & YES & YES \\
\hline 10 & $\mathrm{a}$ & 106.57 & 0.00000 & NO & YES \\
\hline 11 & $\mathrm{~b} 2$ & 175.07 & 0.02567 & YES & YES \\
\hline 12 & b3 & 175.27 & 0.02479 & YES & YES \\
\hline 13 & $\mathrm{~b} 2$ & 211.94 & 3.45683 & YES & YES \\
\hline 14 & b3 & 212.11 & 3.45005 & YES & YES \\
\hline 15 & $a$ & 225.23 & 0.00000 & NO & YES \\
\hline 16 & $\mathrm{~b} 1$ & 238.59 & 0.00044 & YES & YES \\
\hline 17 & $\mathrm{~b} 1$ & 277.58 & 49.14905 & YES & YES \\
\hline 18 & $\mathrm{~b} 1$ & 277.83 & 17.46302 & YES & YES \\
\hline 19 & $\mathrm{~b} 2$ & 288.57 & 6.00415 & YES & YES \\
\hline 20 & b3 & 288.60 & 6.04661 & YES & YES \\
\hline 21 & b3 & 328.77 & 8.60578 & YES & YES \\
\hline 22 & $\mathrm{~b} 2$ & 328.87 & 8.62934 & YES & YES \\
\hline 23 & b1 & 378.15 & 306.76861 & YES & YES \\
\hline 24 & $\mathrm{~b} 1$ & 469.00 & 0.00002 & YES & YES \\
\hline 25 & $a$ & 469.43 & 0.00000 & NO & YES \\
\hline 26 & $\mathrm{a}$ & 523.07 & 0.00000 & NO & YES \\
\hline 27 & a & 531.60 & 0.00000 & NO & YES \\
\hline 28 & b3 & 536.75 & 48.04416 & YES & YES \\
\hline 29 & $\mathrm{~b} 2$ & 536.75 & 47.68197 & YES & YES \\
\hline 30 & $\mathrm{~b} 2$ & 556.26 & 148.33244 & YES & YES \\
\hline 31 & b3 & 556.31 & 148.20594 & YES & YES \\
\hline 32 & b3 & 670.38 & 0.74046 & YES & YES \\
\hline 33 & $\mathrm{~b} 2$ & 670.39 & 0.74520 & YES & YES \\
\hline 34 & $\mathrm{~b} 1$ & 724.17 & 4.76820 & YES & YES \\
\hline 35 & $a$ & 724.60 & 0.00000 & NO & YES \\
\hline 36 & b3 & 734.41 & 21.29428 & YES & YES \\
\hline 37 & $\mathrm{~b} 2$ & 734.42 & 21.14649 & YES & YES \\
\hline 38 & $\mathrm{~b} 2$ & 870.28 & 73.64902 & YES & YES \\
\hline 39 & b3 & 870.29 & 74.17605 & YES & YES \\
\hline 40 & b1 & 985.14 & 0.00000 & YES & YES \\
\hline 41 & $\mathrm{a}$ & 985.70 & 0.00000 & NO & YES \\
\hline 42 & $a$ & 1063.46 & 0.00000 & $\mathrm{NO}$ & YES \\
\hline 43 & b1 & 1063.58 & 6.20602 & YES & YES \\
\hline 44 & $\mathrm{~b} 2$ & 1079.37 & 0.77343 & YES & YES \\
\hline 45 & b3 & 1079.40 & 0.78361 & YES & YES \\
\hline 46 & $\mathrm{~b} 1$ & 1086.19 & 41.46501 & YES & YES \\
\hline 47 & $\mathrm{a}$ & 1093.33 & 0.00000 & NO & YES \\
\hline 48 & $\mathrm{~b} 2$ & 1114.79 & 8.60958 & YES & YES \\
\hline 49 & b3 & 1114.79 & 8.56115 & YES & YES \\
\hline 50 & $\mathrm{~b} 2$ & 1133.15 & 0.01753 & YES & YES \\
\hline 51 & b3 & 1133.15 & 0.01711 & YES & YES \\
\hline 52 & $\mathrm{~b} 1$ & 1136.10 & 0.00004 & YES & YES \\
\hline 53 & $\mathrm{a}$ & 1136.77 & 0.00000 & NO & YES \\
\hline 54 & $\mathrm{~b} 1$ & 1142.81 & 0.30050 & YES & YES \\
\hline 55 & $a$ & 1146.18 & 0.00000 & NO & YES \\
\hline 56 & b3 & 1180.23 & 3.22688 & YES & YES \\
\hline 57 & $\mathrm{~b} 2$ & 1180.24 & 3.24984 & YES & YES \\
\hline 58 & b1 & 1299.05 & 12.66855 & YES & YES \\
\hline 59 & a & 1299.79 & 0.00000 & NO & YES \\
\hline 60 & b3 & 1326.97 & 0.31808 & YES & YES \\
\hline 61 & $\mathrm{~b} 2$ & 1326.97 & 0.31972 & YES & YES \\
\hline
\end{tabular}




$\begin{array}{llrrll}62 & \text { b2 } & 1476.02 & 8.94498 & \text { YES } & \text { YES } \\ 63 & \text { b3 } & 1476.02 & 8.88168 & \text { YES } & \text { YES } \\ 64 & \text { b3 } & 1584.03 & 25.41726 & \text { YES } & \text { YES } \\ 65 & \text { b2 } & 1584.03 & 25.59953 & \text { YES } & \text { YES } \\ 66 & \text { b1 } & 1633.86 & 44.54449 & \text { YES } & \text { YES } \\ 67 & \text { a } & 1633.94 & 0.00000 & \text { NO } & \text { YES } \\ 68 & \text { b1 } & 1724.78 & 5.09110 & \text { YES } & \text { YES } \\ 69 & \text { a } & 1726.87 & 0.00000 & \text { NO } & \text { YES } \\ 70 & \text { b3 } & 1727.86 & 0.27775 & \text { YES } & \text { YES } \\ 71 & \text { b2 } & 1727.87 & 0.27804 & \text { YES } & \text { YES } \\ 72 & \text { b1 } & 3255.10 & 0.27273 & \text { YES } & \text { YES } \\ 73 & \text { a } & 3255.12 & 0.00000 & \text { NO } & \text { YES } \\ 74 & \text { b3 } & 3265.84 & 11.02671 & \text { YES } & \text { YES } \\ 75 & \text { b2 } & 3265.84 & 11.11902 & \text { YES } & \text { YES } \\ 76 & \text { b1 } & 3278.78 & 23.06520 & \text { YES } & \text { YES } \\ 77 & \text { a } & 3278.86 & 0.00000 & \text { NO } & \text { YES } \\ 78 & \text { b3 } & 3296.02 & 0.00354 & \text { YES } & \text { YES } \\ 79 & \text { b2 } & 3296.02 & 0.00396 & \text { YES } & \text { YES } \\ 80 & \text { b1 } & 3296.30 & 16.08415 & \text { YES } & \text { YES } \\ 81 & \text { a } & 3296.38 & 0.00000 & \text { NO } & \text { YES }\end{array}$



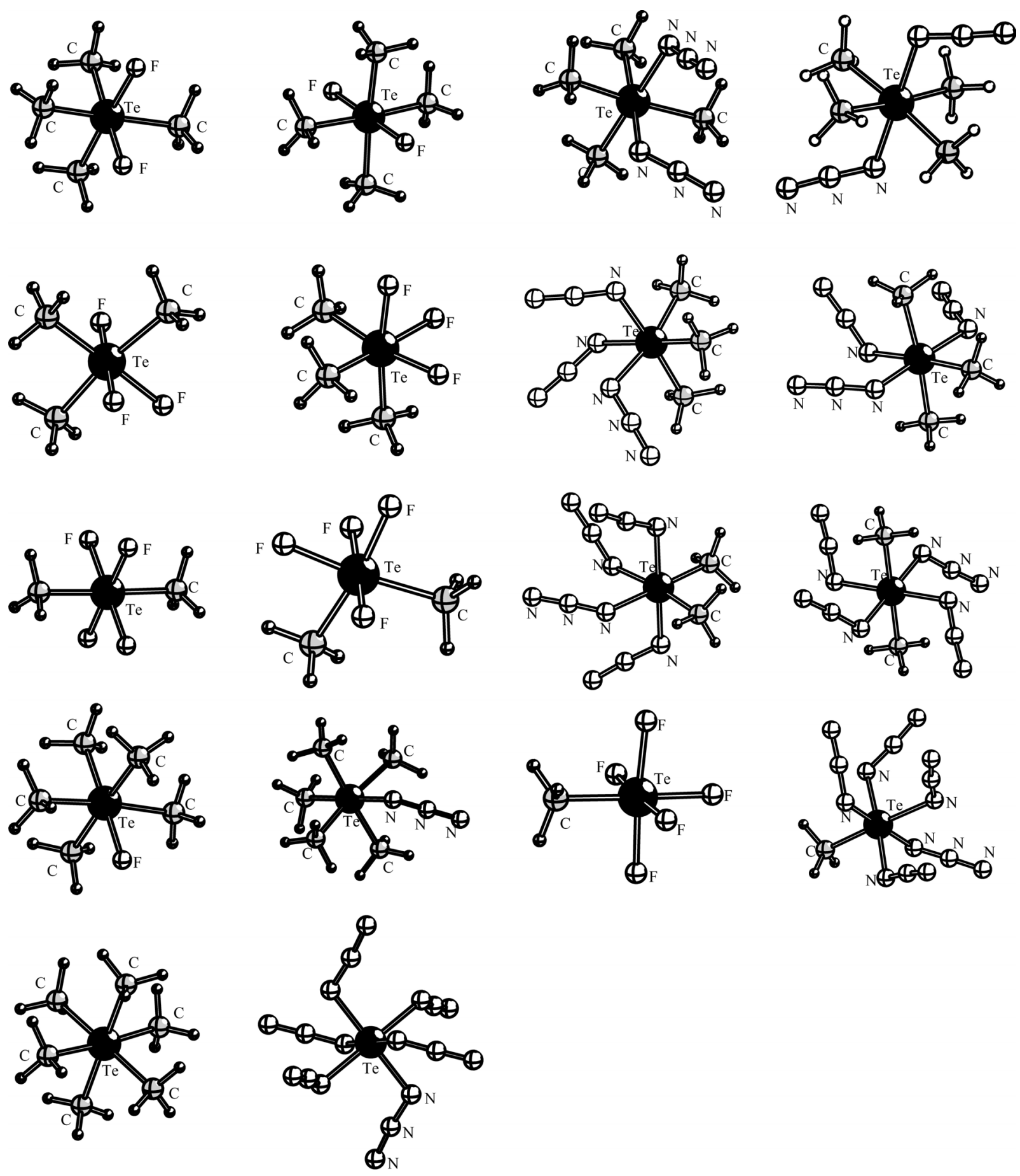

Calculated molecular structures for the $\mathrm{Me}_{\mathrm{x}} \mathrm{TeF}_{6-\mathrm{x}}$ and $\mathrm{Me}_{\mathrm{x}} \mathrm{Te}\left(\mathrm{N}_{3}\right)_{6-\mathrm{x}}(\mathrm{x}=0-6)$ molecules at the MP2/cc-pVDZ level of theory. 
Cartesian coordinates of the MP2(FC)/cc-pVDZ $(\mathrm{PP}(\mathrm{Te})=\mathrm{MDF} 28)$ minimum structures for the $\mathrm{Me}_{\mathrm{x}} \mathrm{TeF}_{6-\mathrm{x}}$ and $\mathrm{Me}_{\mathrm{x}} \mathrm{Te}\left(\mathrm{N}_{3}\right)_{6-\mathrm{x}}(\mathrm{x}=0-6)$ molecules:

\begin{tabular}{|c|c|c|c|}
\hline \multicolumn{4}{|l|}{13} \\
\hline \multicolumn{4}{|c|}{ cis-me2tef 4} \\
\hline $\mathrm{Te}$ & 1.88177 & 2.51668 & 1.90595 \\
\hline F & 2.01563 & 2.49501 & 3.82575 \\
\hline $\mathrm{C}$ & 3.97332 & 2.47601 & 1.75018 \\
\hline $\mathrm{H}$ & 4.22632 & 2.49122 & 0.68017 \\
\hline $\mathrm{H}$ & 4.35861 & 3.36959 & 2.25962 \\
\hline $\mathrm{H}$ & 4.33563 & 1.55827 & 2.23659 \\
\hline $\mathrm{F}$ & 1.97849 & 4.42835 & 1.96386 \\
\hline F & 1.65286 & 2.65457 & 0.00000 \\
\hline $\mathrm{C}$ & 1.49479 & 0.45707 & 1.81265 \\
\hline $\mathrm{H}$ & 1.57507 & 0.05804 & 2.83419 \\
\hline $\mathrm{H}$ & 0.47752 & 0.33034 & 1.41820 \\
\hline $\mathrm{H}$ & 2.23719 & 0.00000 & 1.14182 \\
\hline F & 0.00000 & 2.80788 & 2.10903 \\
\hline \multicolumn{4}{|l|}{21} \\
\hline \multicolumn{4}{|c|}{ cis-me 2 ten 12} \\
\hline $\mathrm{Te}$ & 3.25538 & 2.56718 & 3.21471 \\
\hline $\mathrm{N}$ & 3.25538 & 2.56718 & 5.33386 \\
\hline $\mathrm{N}$ & 5.37575 & 2.56718 & 3.17831 \\
\hline $\mathrm{N}$ & 1.13501 & 2.56718 & 3.25111 \\
\hline $\mathrm{N}$ & 3.25538 & 2.56718 & 1.09556 \\
\hline $\mathrm{N}$ & 5.91532 & 1.82500 & 4.01379 \\
\hline $\mathrm{N}$ & 3.88805 & 1.64051 & 0.57064 \\
\hline $\mathrm{N}$ & 0.59544 & 3.30935 & 2.41563 \\
\hline $\mathrm{N}$ & 2.62270 & 3.49385 & 5.85878 \\
\hline $\mathrm{N}$ & 6.51076 & 1.15030 & 4.76903 \\
\hline $\mathrm{N}$ & 2.04481 & 4.34301 & 6.42942 \\
\hline $\mathrm{N}$ & 0.00000 & 3.98405 & 1.66040 \\
\hline $\mathrm{N}$ & 4.46595 & 0.79134 & 0.00000 \\
\hline $\mathrm{C}$ & 3.18917 & 0.44260 & 3.39624 \\
\hline $\mathrm{H}$ & 3.80789 & 0.18899 & 4.26971 \\
\hline $\mathrm{H}$ & 2.12747 & 0.20794 & 3.55697 \\
\hline $\mathrm{H}$ & 3.58544 & 0.00000 & 2.46990 \\
\hline C & 3.32158 & 4.69175 & 3.03319 \\
\hline $\mathrm{H}$ & 4.38329 & 4.92641 & 2.87246 \\
\hline $\mathrm{H}$ & 2.70287 & 4.94536 & 2.15972 \\
\hline $\mathrm{H}$ & 2.92532 & 5.13435 & 3.95952 \\
\hline \multicolumn{4}{|c|}{$\begin{array}{l}19 \\
\text { cis-me4tef } 2\end{array}$} \\
\hline $\mathrm{Te}$ & 2.37481 & 2.28099 & 2.63779 \\
\hline $\mathrm{C}$ & 2.30898 & 2.33016 & 4.76038 \\
\hline $\mathrm{H}$ & 3.26826 & 2.73853 & 5.10697 \\
\hline $\mathrm{H}$ & 2.18163 & 1.29566 & 5.11032 \\
\hline $\mathrm{H}$ & 1.47051 & 2.96662 & 5.08435 \\
\hline $\mathrm{C}$ & 1.24920 & 4.08034 & 2.37789 \\
\hline $\mathrm{H}$ & 1.91921 & 4.92631 & 2.59604 \\
\hline $\mathrm{H}$ & 0.38945 & 4.08619 & 3.06705 \\
\hline $\mathrm{H}$ & 0.89132 & 4.14514 & 1.33748 \\
\hline $\mathrm{C}$ & 2.90748 & 2.13998 & 0.58634 \\
\hline $\mathrm{H}$ & 3.27949 & 3.12628 & 0.27377 \\
\hline $\mathrm{H}$ & 2.02365 & 1.84316 & 0.00000 \\
\hline $\mathrm{H}$ & 3.70242 & 1.38575 & 0.50690 \\
\hline
\end{tabular}




$\begin{array}{llll}\mathrm{C} & 0.63886 & 1.04500 & 2.46171 \\ \mathrm{H} & 0.97957 & 0.00000 & 2.39800 \\ \mathrm{H} & 0.07523 & 1.31911 & 1.55544 \\ \mathrm{H} & 0.00000 & 1.17674 & 3.35010 \\ \mathrm{~F} & 4.03259 & 3.35757 & 2.79365 \\ \mathrm{~F} & 3.48471 & 0.65959 & 2.90370 \\ \mathrm{H} & & & \\ \mathrm{Ci} & & & \\ \mathrm{Te} & 2.62656 & 2.59150 & 2.56651 \\ \mathrm{~N} & 2.61244 & 2.59264 & 4.78130 \\ \mathrm{C} & 4.78683 & 2.66934 & 2.51524 \\ \mathrm{H} & 5.02105 & 3.51147 & 1.84747 \\ \mathrm{H} & 5.20279 & 2.84668 & 3.51861 \\ \mathrm{H} & 5.14486 & 1.71079 & 2.10647 \\ \mathrm{C} & 2.51395 & 2.69178 & 0.43360 \\ \mathrm{H} & 2.60973 & 3.74974 & 0.14456 \\ \mathrm{H} & 3.33442 & 2.09752 & 0.00000 \\ \mathrm{H} & 1.54290 & 2.29034 & 0.10171 \\ \mathrm{C} & 0.51497 & 2.85929 & 2.82843 \\ \mathrm{H} & 0.24037 & 3.74552 & 2.23768 \\ \mathrm{H} & 0.00000 & 1.95270 & 2.47013 \\ \mathrm{H} & 0.34348 & 3.02397 & 3.90174 \\ \mathrm{C} & 2.49007 & 0.44997 & 2.63972 \\ \mathrm{H} & 2.09124 & 0.18751 & 3.63117 \\ \mathrm{H} & 1.80928 & 0.10728 & 1.84311 \\ \mathrm{H} & 3.48597 & 0.00000 & 2.50340 \\ \mathrm{~N} & 3.67164 & 2.75051 & 5.38838 \\ \mathrm{~N} & 4.65942 & 2.89508 & 6.02423 \\ \mathrm{~N} & 2.72931 & 4.78599 & 2.53712 \\ \mathrm{~N} & 2.95474 & 5.32202 & 3.62849 \\ \mathrm{~N} & 3.17289 & 5.90272 & 4.63260\end{array}$

16

fac-me 3 tef 3

$\begin{array}{rlll}\mathrm{Te} & 2.61603 & 2.51645 & 1.91637 \\ \mathrm{C} & 2.58647 & 2.49699 & 4.03017 \\ \mathrm{H} & 3.62682 & 2.41697 & 4.37736 \\ \mathrm{H} & 2.14250 & 3.44248 & 4.37416 \\ \mathrm{H} & 1.99389 & 1.63479 & 4.37528 \\ \mathrm{C} & 0.53431 & 2.77677 & 1.65541 \\ \mathrm{H} & 0.26200 & 2.38055 & 0.66635 \\ \mathrm{H} & 0.00000 & 2.24029 & 2.45562 \\ \mathrm{H} & 0.32458 & 3.85516 & 1.70389 \\ \mathrm{~F} & 2.82156 & 2.74091 & 0.00000 \\ \mathrm{~F} & 2.83875 & 4.44224 & 1.99904 \\ \mathrm{C} & 2.62317 & 0.41761 & 1.66296 \\ \mathrm{H} & 3.50459 & 0.01404 & 2.18219 \\ \mathrm{H} & 2.68994 & 0.21223 & 0.58470 \\ \mathrm{H} & 1.69365 & 0.00000 & 2.08159 \\ \mathrm{~F} & 4.55519 & 2.45668 & 1.95056\end{array}$

22

fac-me 3 ten 9

$\begin{array}{rlll}\mathrm{Te} & 2.51203 & 3.37659 & 2.56200 \\ \mathrm{~N} & 2.51203 & 3.37659 & 4.71129 \\ \mathrm{~N} & 4.64851 & 3.37659 & 2.64251 \\ \mathrm{~N} & 2.76642 & 1.19688 & 2.68782 \\ \mathrm{~N} & 1.90087 & 0.60492 & 2.03848 \\ \mathrm{~N} & 2.41139 & 2.27959 & 5.28251 \\ \mathrm{~N} & 5.12535 & 2.70072 & 3.56598\end{array}$




$\begin{array}{llll}\mathrm{N} & 5.64992 & 2.08529 & 4.41531 \\ \mathrm{~N} & 1.09520 & 0.00000 & 1.41956 \\ \mathrm{~N} & 2.30832 & 1.29791 & 5.91911 \\ \mathrm{C} & 2.66994 & 3.25119 & 0.43731 \\ \mathrm{H} & 2.09178 & 2.37841 & 0.09387 \\ \mathrm{H} & 2.26282 & 4.17744 & 0.00000 \\ \mathrm{H} & 3.73724 & 3.13447 & 0.20058 \\ \mathrm{C} & 0.36979 & 3.26389 & 2.57925 \\ \mathrm{H} & 0.04399 & 3.64866 & 3.55697 \\ \mathrm{H} & 0.00000 & 3.89967 & 1.75782 \\ \mathrm{H} & 0.03130 & 2.22655 & 2.43172 \\ \mathrm{C} & 2.58765 & 5.49366 & 2.69079 \\ \mathrm{H} & 3.52832 & 5.81519 & 2.21991 \\ \mathrm{H} & 1.71334 & 5.91809 & 2.17173 \\ \mathrm{H} & 2.57915 & 5.75422 & 3.75953\end{array}$

$$
22
$$

$\begin{array}{cccc}\mathrm{Te} & 2.62754 & 2.60449 & 2.55314 \\ \mathrm{~N} & 2.56011 & 2.57490 & 4.79642 \\ \mathrm{C} & 4.79164 & 2.66382 & 2.78220 \\ \mathrm{H} & 5.06972 & 3.62906 & 3.23324 \\ \mathrm{H} & 5.08762 & 1.84052 & 3.45293 \\ \mathrm{H} & 5.27249 & 2.54212 & 1.79711 \\ \mathrm{C} & 2.58209 & 2.72354 & 0.40215 \\ \mathrm{H} & 3.60786 & 2.72524 & 0.00000 \\ \mathrm{H} & 2.03430 & 1.85169 & 0.00917 \\ \mathrm{H} & 2.06703 & 3.65066 & 0.10281 \\ \mathrm{C} & 0.46913 & 2.52008 & 2.74846 \\ \mathrm{H} & 0.00000 & 2.73581 & 1.77304 \\ \mathrm{H} & 0.18091 & 1.51958 & 3.10611 \\ \mathrm{H} & 0.18227 & 3.27503 & 3.49625 \\ \mathrm{C} & 2.54653 & 4.76528 & 2.74057 \\ \mathrm{H} & 1.58742 & 5.12720 & 2.33447 \\ \mathrm{H} & 2.63110 & 5.01071 & 3.81058 \\ \mathrm{H} & 3.38708 & 5.19883 & 2.17334\end{array}$




\begin{tabular}{|c|c|c|c|}
\hline C & 2.75670 & 0.42954 & 2.45207 \\
\hline $\mathrm{H}$ & 2.95620 & 0.00000 & 3.44739 \\
\hline $\mathrm{H}$ & 1.80895 & 0.03918 & 2.04677 \\
\hline $\mathrm{H}$ & 3.59018 & 0.18497 & 1.77166 \\
\hline N & 3.21511 & 1.66573 & 5.30055 \\
\hline $\mathrm{N}$ & 3.83920 & 0.79600 & 5.81341 \\
\hline \multicolumn{4}{|c|}{$\begin{array}{l}25 \\
\text { me6t }\end{array}$} \\
\hline $\mathrm{Te}$ & 2.60957 & 2.65409 & 2.62643 \\
\hline $\mathrm{C}$ & 2.56396 & 2.63992 & 4.81797 \\
\hline $\mathrm{H}$ & 3.57821 & 2.49097 & 5.22474 \\
\hline $\mathrm{H}$ & 2.17285 & 3.61410 & 5.15807 \\
\hline $\mathrm{H}$ & 1.89962 & 1.83371 & 5.17283 \\
\hline $\mathrm{C}$ & 3.43244 & 4.68291 & 2.73554 \\
\hline $\mathrm{H}$ & 3.89027 & 4.95311 & 1.76878 \\
\hline $\mathrm{H}$ & 2.64051 & 5.40827 & 2.98668 \\
\hline $\mathrm{H}$ & 4.20450 & 4.69672 & 3.52381 \\
\hline $\mathrm{C}$ & 0.53595 & 3.36461 & 2.60696 \\
\hline $\mathrm{H}$ & 0.00000 & 2.83298 & 1.80211 \\
\hline $\mathrm{H}$ & 0.05597 & 3.14374 & 3.57550 \\
\hline $\mathrm{H}$ & 0.50104 & 4.45002 & 2.41451 \\
\hline $\mathrm{C}$ & 2.59590 & 2.79793 & 0.43914 \\
\hline $\mathrm{H}$ & 3.63108 & 2.79148 & 0.05756 \\
\hline $\mathrm{H}$ & 2.03329 & 1.95710 & 0.00000 \\
\hline $\mathrm{H}$ & 2.10861 & 3.74845 & 0.16215 \\
\hline C & 1.88116 & 0.58662 & 2.61698 \\
\hline $\mathrm{H}$ & 0.83237 & 0.55814 & 2.95826 \\
\hline $\mathrm{H}$ & 1.95366 & 0.15593 & 1.60425 \\
\hline $\mathrm{H}$ & 2.50856 & 0.00000 & 3.30972 \\
\hline $\mathrm{C}$ & 4.64807 & 1.85254 & 2.54189 \\
\hline $\mathrm{H}$ & 4.95119 & 1.46234 & 3.52790 \\
\hline $\mathrm{H}$ & 4.66256 & 1.03244 & 1.80373 \\
\hline $\mathrm{H}$ & 5.34815 & 2.64407 & 2.22471 \\
\hline \multicolumn{4}{|l|}{16} \\
\hline \multicolumn{4}{|c|}{ mer-me3tef 3} \\
\hline $\mathrm{Te}$ & 2.45059 & 2.08257 & 1.96016 \\
\hline $\mathrm{C}$ & 2.48463 & 2.02448 & 4.08241 \\
\hline $\mathrm{H}$ & 3.53102 & 2.01672 & 4.42365 \\
\hline $\mathrm{H}$ & 1.97981 & 1.10788 & 4.42365 \\
\hline $\mathrm{H}$ & 1.96111 & 2.91801 & 4.45676 \\
\hline $\mathrm{C}$ & 0.61545 & 1.07533 & 1.76845 \\
\hline $\mathrm{H}$ & 0.14347 & 1.42482 & 0.84121 \\
\hline $\mathrm{H}$ & 0.00000 & 1.31809 & 2.64701 \\
\hline $\mathrm{H}$ & 0.84031 & 0.00000 & 1.71804 \\
\hline $\mathrm{F}$ & 2.47204 & 2.04597 & 0.00000 \\
\hline $\mathrm{C}$ & 4.22645 & 3.19100 & 1.76845 \\
\hline $\mathrm{H}$ & 4.31558 & 3.84656 & 2.64701 \\
\hline $\mathrm{H}$ & 5.05457 & 2.46911 & 1.71804 \\
\hline $\mathrm{H}$ & 4.15234 & 3.77359 & 0.84121 \\
\hline $\mathrm{F}$ & 1.46331 & 3.76766 & 1.95555 \\
\hline $\mathrm{F}$ & 3.43696 & 0.39905 & 1.94984 \\
\hline \multicolumn{3}{|l|}{22} & mer-me3ten 9 \\
\hline $\mathrm{Te}$ & 3.31238 & 2.49819 & 2.54068 \\
\hline $\mathrm{N}$ & 3.30865 & 2.44639 & 4.73310 \\
\hline $\mathrm{N}$ & 5.44302 & 2.51750 & 2.45828 \\
\hline $\mathrm{N}$ & 1.18409 & 2.48463 & 2.47100 \\
\hline $\mathrm{N}$ & 6.01003 & 2.75833 & 3.53543 \\
\hline
\end{tabular}




\begin{tabular}{|c|c|c|c|}
\hline $\mathrm{N}$ & 0.61906 & 2.71922 & 3.55099 \\
\hline $\mathrm{N}$ & 3.27625 & 3.49993 & 5.37330 \\
\hline $\mathrm{N}$ & 6.62999 & 2.98445 & 4.50770 \\
\hline $\mathrm{N}$ & 3.24967 & 4.47401 & 6.04319 \\
\hline $\mathrm{N}$ & 0.00000 & 2.93781 & 4.52519 \\
\hline $\mathrm{C}$ & 3.36637 & 0.37754 & 2.79544 \\
\hline $\mathrm{H}$ & 3.58824 & 0.18533 & 3.85430 \\
\hline $\mathrm{H}$ & 2.37400 & 0.00000 & 2.50555 \\
\hline $\mathrm{H}$ & 4.16529 & 0.01251 & 2.13221 \\
\hline $\mathrm{C}$ & 3.27829 & 2.37320 & 0.41734 \\
\hline $\mathrm{H}$ & 4.30115 & 2.14726 & 0.07862 \\
\hline $\mathrm{H}$ & 2.57904 & 1.56941 & 0.14032 \\
\hline $\mathrm{H}$ & 2.93107 & 3.33039 & 0.00000 \\
\hline $\mathrm{C}$ & 3.32185 & 4.65453 & 2.52227 \\
\hline $\mathrm{H}$ & 4.17899 & 4.91857 & 1.88446 \\
\hline $\mathrm{H}$ & 2.35901 & 4.94612 & 2.07581 \\
\hline $\mathrm{H}$ & 3.43548 & 5.06856 & 3.53496 \\
\hline \multicolumn{4}{|c|}{$\begin{array}{l}10 \\
\text { metef } 5\end{array}$} \\
\hline $\mathrm{Te}$ & 2.44154 & 1.89948 & 1.89400 \\
\hline $\mathrm{F}$ & 2.42515 & 1.81580 & 3.79603 \\
\hline $\mathrm{F}$ & 4.32437 & 1.92376 & 1.99227 \\
\hline $\mathrm{F}$ & 2.62130 & 1.97262 & 0.00000 \\
\hline $\mathrm{C}$ & 0.36396 & 1.90658 & 1.78433 \\
\hline $\mathrm{H}$ & 0.09669 & 2.49299 & 0.89507 \\
\hline $\mathrm{H}$ & 0.04494 & 0.85912 & 1.69695 \\
\hline $\mathrm{H}$ & 0.00000 & 2.37061 & 2.71080 \\
\hline $\mathrm{F}$ & 2.49479 & 3.80083 & 1.97585 \\
\hline $\mathrm{F}$ & 2.55121 & 0.00000 & 1.82165 \\
\hline \multicolumn{4}{|c|}{$\begin{array}{l}20 \\
\text { meten15 }\end{array}$} \\
\hline $\mathrm{Te}$ & 3.23960 & 2.44693 & 3.29052 \\
\hline $\mathrm{N}$ & 3.23960 & 2.44693 & 5.36868 \\
\hline $\mathrm{N}$ & 5.33477 & 2.44693 & 3.25666 \\
\hline $\mathrm{N}$ & 3.18345 & 4.58348 & 3.48105 \\
\hline $\mathrm{N}$ & 1.15234 & 2.35384 & 3.22953 \\
\hline $\mathrm{N}$ & 3.13135 & 2.57091 & 1.17898 \\
\hline $\mathrm{N}$ & 5.89649 & 3.54310 & 3.43577 \\
\hline $\mathrm{N}$ & 4.18530 & 2.92759 & 0.61935 \\
\hline $\mathrm{N}$ & 3.20403 & 5.20903 & 2.40593 \\
\hline $\mathrm{N}$ & 0.60369 & 3.39140 & 2.81361 \\
\hline $\mathrm{N}$ & 2.57876 & 3.35524 & 5.90760 \\
\hline $\mathrm{N}$ & 6.52264 & 4.51992 & 3.59129 \\
\hline $\mathrm{N}$ & 1.98622 & 4.15409 & 6.52183 \\
\hline $\mathrm{N}$ & 0.00000 & 4.31865 & 2.43350 \\
\hline $\mathrm{N}$ & 5.12383 & 3.26033 & 0.00000 \\
\hline $\mathrm{N}$ & 3.23147 & 5.88433 & 1.44604 \\
\hline $\mathrm{C}$ & 3.33294 & 0.34412 & 3.26381 \\
\hline $\mathrm{H}$ & 3.45164 & 0.01736 & 4.30712 \\
\hline $\mathrm{H}$ & 2.38672 & 0.00000 & 2.82297 \\
\hline $\mathrm{H}$ & 4.20348 & 0.08125 & 2.64617 \\
\hline \multicolumn{4}{|l|}{$\begin{array}{l}7 \\
\text { tef }\end{array}$} \\
\hline $\mathrm{Te}$ & 1.88011 & 1.88011 & 1.88011 \\
\hline $\mathrm{F}$ & 1.88011 & 1.88011 & 0.00000 \\
\hline $\mathrm{F}$ & 0.00000 & 1.88011 & 1.88011 \\
\hline $\mathrm{F}$ & 1.88011 & 3.76021 & 1.88011 \\
\hline
\end{tabular}




\begin{tabular}{|c|c|c|c|}
\hline $\mathrm{F}$ & 3.76021 & 1.88011 & 1.88011 \\
\hline $\mathrm{F}$ & 1.88011 & 0.00000 & 1.88011 \\
\hline $\mathrm{F}$ & 1.88011 & 1.88011 & 3.76021 \\
\hline \multicolumn{4}{|c|}{$\begin{array}{l}19 \\
\text { ten18 }\end{array}$} \\
\hline $\mathrm{Te}$ & 2.96541 & 3.26119 & 3.21285 \\
\hline $\mathrm{N}$ & 2.91585 & 3.25456 & 5.29238 \\
\hline $\mathrm{N}$ & 4.04501 & 3.33267 & 5.82000 \\
\hline $\mathrm{N}$ & 4.26025 & 1.63722 & 3.32749 \\
\hline $\mathrm{N}$ & 1.28413 & 2.03745 & 3.25816 \\
\hline $\mathrm{N}$ & 3.01496 & 3.26783 & 1.13332 \\
\hline $\mathrm{N}$ & 4.64669 & 4.48493 & 3.16754 \\
\hline $\mathrm{N}$ & 1.67057 & 4.88517 & 3.09821 \\
\hline $\mathrm{N}$ & 1.43941 & 0.99842 & 3.93329 \\
\hline $\mathrm{N}$ & 0.83203 & 4.92251 & 4.02287 \\
\hline $\mathrm{N}$ & 1.88581 & 3.18971 & 0.60570 \\
\hline $\mathrm{N}$ & 4.49141 & 5.52396 & 2.49241 \\
\hline $\mathrm{N}$ & 5.09879 & 1.59988 & 2.40283 \\
\hline $\mathrm{N}$ & 1.46270 & 0.00000 & 4.53867 \\
\hline $\mathrm{N}$ & 5.04155 & 3.39545 & 6.42570 \\
\hline $\mathrm{N}$ & 5.93082 & 1.46153 & 1.59507 \\
\hline $\mathrm{N}$ & 4.46812 & 6.52239 & 1.88703 \\
\hline $\mathrm{N}$ & 0.00000 & 5.06086 & 4.83064 \\
\hline $\mathrm{N}$ & 0.88927 & 3.12694 & 0.00000 \\
\hline \multicolumn{4}{|l|}{13} \\
\hline $\mathrm{Te}$ & 1.57091 & 1.56828 & 2.41405 \\
\hline $\mathrm{C}$ & 1.57028 & 1.59053 & 4.49319 \\
\hline $\mathrm{H}$ & 2.56629 & 1.25933 & 4.81546 \\
\hline $\mathrm{H}$ & 0.78321 & 0.89791 & 4.81960 \\
\hline $\mathrm{H}$ & 1.36105 & 2.62436 & 4.79712 \\
\hline $\mathrm{F}$ & 2.72044 & 3.12477 & 2.42420 \\
\hline $\mathrm{C}$ & 1.57016 & 1.61768 & 0.33537 \\
\hline $\mathrm{H}$ & 2.56613 & 1.29062 & 0.00875 \\
\hline $\mathrm{H}$ & 0.78301 & 0.92945 & 0.00000 \\
\hline $\mathrm{H}$ & 1.36101 & 2.65540 & 0.04499 \\
\hline $\mathrm{F}$ & 3.12581 & 0.41705 & 2.40649 \\
\hline $\mathrm{F}$ & 0.00000 & 2.69796 & 2.42146 \\
\hline$F$ & 0.43803 & 0.00000 & 2.40383 \\
\hline \multicolumn{4}{|l|}{21} \\
\hline \multicolumn{4}{|c|}{ trans-me 2 ten 12} \\
\hline $\mathrm{Te}$ & 3.13441 & 2.51463 & 2.46405 \\
\hline $\mathrm{N}$ & 3.13441 & 2.51463 & 4.61512 \\
\hline $\mathrm{N}$ & 5.25134 & 2.51463 & 2.39497 \\
\hline $\mathrm{N}$ & 3.08337 & 4.67529 & 2.51191 \\
\hline $\mathrm{N}$ & 1.04522 & 2.65105 & 2.41766 \\
\hline $\mathrm{N}$ & 5.84089 & 2.64156 & 3.48201 \\
\hline $\mathrm{N}$ & 4.16476 & 5.20726 & 2.81329 \\
\hline $\mathrm{N}$ & 0.55082 & 3.31556 & 3.34426 \\
\hline $\mathrm{N}$ & 3.23703 & 3.61334 & 5.18657 \\
\hline $\mathrm{N}$ & 6.48559 & 2.76073 & 4.45470 \\
\hline $\mathrm{N}$ & 3.34018 & 4.60192 & 5.81273 \\
\hline $\mathrm{N}$ & 0.00000 & 3.92123 & 4.18053 \\
\hline $\mathrm{N}$ & 5.14600 & 5.79110 & 3.09343 \\
\hline $\mathrm{C}$ & 3.07278 & 0.40176 & 2.64754 \\
\hline $\mathrm{H}$ & 3.24926 & 0.15934 & 3.70548 \\
\hline $\mathrm{H}$ & 2.07304 & 0.07549 & 2.32283 \\
\hline $\mathrm{H}$ & 3.86925 & 0.00000 & 2.00284 \\
\hline
\end{tabular}




\begin{tabular}{|c|c|c|c|}
\hline $\mathrm{C}$ & 3.12922 & 2.57434 & 0.34759 \\
\hline $\mathrm{H}$ & 4.15536 & 2.36507 & 0.00946 \\
\hline $\mathrm{H}$ & 2.41604 & 1.81099 & 0.00000 \\
\hline $\mathrm{H}$ & 2.80215 & 3.58240 & 0.05719 \\
\hline \multicolumn{4}{|c|}{$\begin{array}{l}19 \\
\text { trans-me4tef } 2\end{array}$} \\
\hline $\mathrm{Te}$ & 1.96347 & 2.50185 & 2.58899 \\
\hline $\mathrm{C}$ & 1.99944 & 2.50154 & 4.73242 \\
\hline $\mathrm{H}$ & 3.05533 & 2.47037 & 5.04714 \\
\hline $\mathrm{H}$ & 1.51179 & 3.41803 & 5.09820 \\
\hline $\mathrm{H}$ & 1.45840 & 1.61580 & 5.09886 \\
\hline C & 2.01716 & 0.35881 & 2.58132 \\
\hline $\mathrm{H}$ & 2.60990 & 0.01305 & 3.44194 \\
\hline $\mathrm{H}$ & 0.97760 & 0.00000 & 2.65542 \\
\hline $\mathrm{H}$ & 2.48061 & 0.01622 & 1.64356 \\
\hline $\mathrm{C}$ & 1.69291 & 2.50914 & 0.46242 \\
\hline $\mathrm{H}$ & 2.69301 & 2.47913 & 0.00000 \\
\hline $\mathrm{H}$ & 1.10467 & 1.62467 & 0.17393 \\
\hline $\mathrm{H}$ & 1.15843 & 3.42689 & 0.17324 \\
\hline$F$ & 3.92695 & 2.44343 & 2.44794 \\
\hline$F$ & 0.00000 & 2.56027 & 2.73005 \\
\hline C & 2.14438 & 4.63791 & 2.57981 \\
\hline $\mathrm{H}$ & 2.62753 & 4.95166 & 1.64190 \\
\hline $\mathrm{H}$ & 2.75645 & 4.94850 & 3.44031 \\
\hline $\mathrm{H}$ & 1.12795 & 5.05790 & 2.65341 \\
\hline \multicolumn{4}{|c|}{$\begin{array}{l}23 \\
\text { trans-me4ten } 6\end{array}$} \\
\hline $\mathrm{Te}$ & 2.62944 & 2.39713 & 2.57375 \\
\hline $\mathrm{C}$ & 2.63544 & 2.40718 & 4.74192 \\
\hline $\mathrm{H}$ & 3.67305 & 2.27602 & 5.08545 \\
\hline $\mathrm{H}$ & 2.25325 & 3.39202 & 5.05297 \\
\hline $\mathrm{H}$ & 1.98664 & 1.60192 & 5.12676 \\
\hline C & 0.71406 & 3.40028 & 2.60526 \\
\hline $\mathrm{H}$ & 0.90981 & 4.47761 & 2.72805 \\
\hline $\mathrm{H}$ & 0.19903 & 3.19470 & 1.65415 \\
\hline $\mathrm{H}$ & 0.12647 & 3.00717 & 3.45095 \\
\hline C & 2.72125 & 2.56563 & 0.41986 \\
\hline $\mathrm{H}$ & 2.15806 & 3.46196 & 0.11731 \\
\hline $\mathrm{H}$ & 3.77792 & 2.65890 & 0.12031 \\
\hline $\mathrm{H}$ & 2.27152 & 1.65158 & 0.00000 \\
\hline $\mathrm{C}$ & 4.44669 & 1.21533 & 2.52744 \\
\hline $\mathrm{H}$ & 4.35683 & 0.53351 & 1.66734 \\
\hline $\mathrm{H}$ & 5.32412 & 1.87370 & 2.40839 \\
\hline $\mathrm{H}$ & 4.50924 & 0.64008 & 3.46394 \\
\hline $\mathrm{N}$ & 3.61305 & 4.30885 & 2.72292 \\
\hline $\mathrm{N}$ & 1.59345 & 0.51902 & 2.36388 \\
\hline $\mathrm{N}$ & 4.70494 & 4.36241 & 2.14805 \\
\hline $\mathrm{N}$ & 0.78419 & 0.28390 & 3.26688 \\
\hline $\mathrm{N}$ & 5.75487 & 4.47552 & 1.62248 \\
\hline $\mathrm{N}$ & 0.00000 & 0.00000 & 4.10104 \\
\hline
\end{tabular}


Analytical frequency analysis of the $\mathrm{Me}_{\mathrm{x}} \mathrm{TeF}_{6-\mathrm{x}}$ and $\mathrm{Me}_{\mathrm{x}} \mathrm{Te}\left(\mathrm{N}_{3}\right)_{6-\mathrm{x}}(\mathrm{x}=0-6)$ molecules at the MP2(FC)/cc-pVDZ (PP(Te) = MDF28) level:

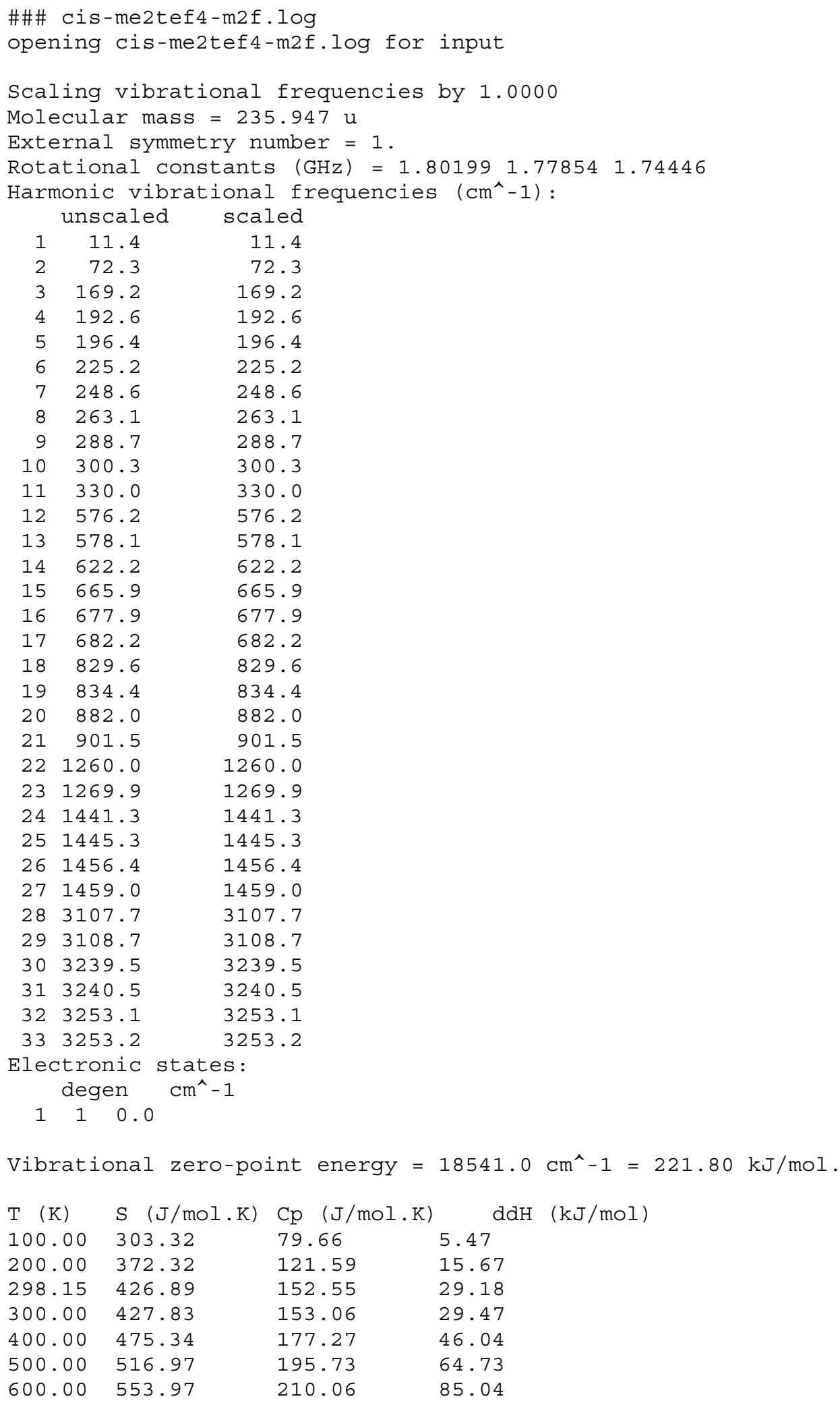




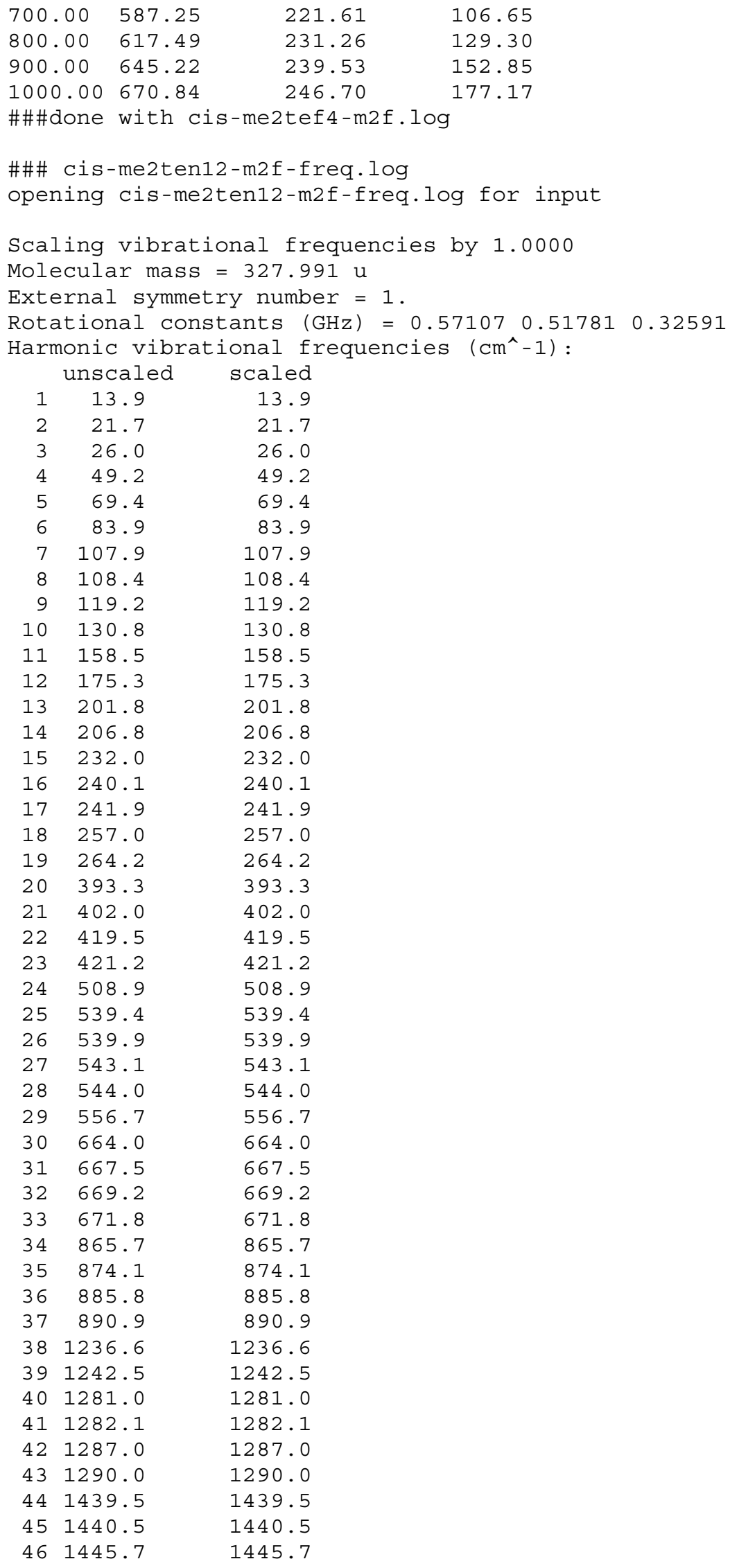




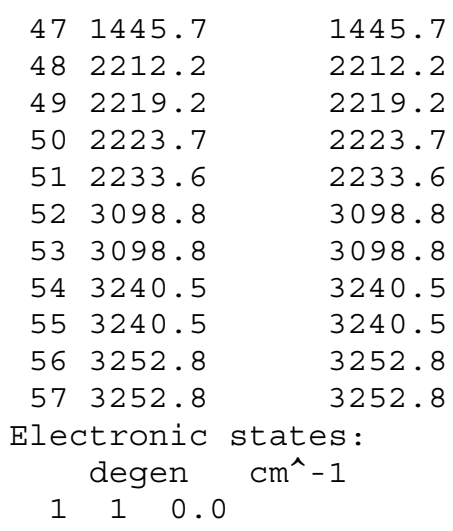




$\begin{array}{rrr}22 & 783.9 & 783.9 \\ 23 & 844.9 & 844.9 \\ 24 & 869.6 & 869.6 \\ 25 & 872.2 & 872.2 \\ 26 & 878.0 & 878.0 \\ 27 & 881.8 & 881.8 \\ 28 & 1239.1 & 1239.1 \\ 29 & 1239.4 & 1239.4 \\ 30 & 1247.2 & 1247.2 \\ 31 & 1263.3 & 1263.3 \\ 32 & 1436.6 & 1436.6 \\ 33 & 1441.1 & 1441.1 \\ 34 & 1450.0 & 1450.0 \\ 35 & 1452.3 & 1452.3 \\ 36 & 1457.5 & 1457.5 \\ 37 & 1460.0 & 1460.0 \\ 38 & 1466.2 & 1466.2 \\ 39 & 1474.5 & 1474.5 \\ 40 & 3086.7 & 3086.7 \\ 41 & 3087.0 & 3087.0 \\ 42 & 3100.4 & 3100.4 \\ 43 & 3100.6 & 3100.6 \\ 44 & 3206.8 & 3206.8 \\ 45 & 3207.8 & 3207.8 \\ 46 & 3221.8 & 3221.8 \\ 47 & 3222.0 & 3222.0 \\ 48 & 3228.3 & 3228.3 \\ 49 & 3228.4 & 3228.4 \\ 50 & 3248.1 & 3248.1 \\ 51 & 3248.2 & 3248.2 \\ \text { Eectronic states } \\ \text { degen }\end{array}$

Vibrational zero-point energy $=33608.5 \mathrm{~cm}^{\wedge}-1=402.05 \mathrm{~kJ} / \mathrm{mol}$.

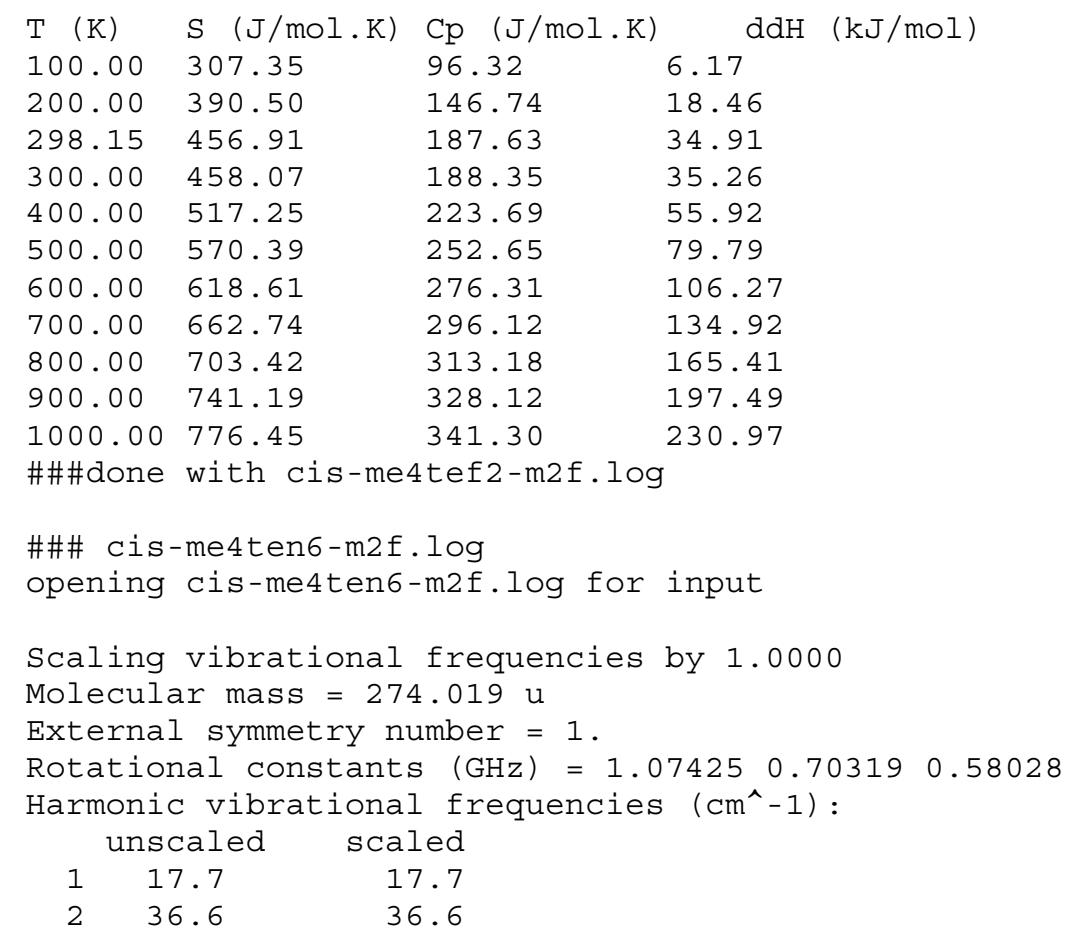




\begin{tabular}{|c|c|c|}
\hline 3 & 54.4 & 54.4 \\
\hline 4 & 94.0 & 94.0 \\
\hline 5 & 101.9 & 101.9 \\
\hline 6 & 108.8 & 108.8 \\
\hline 7 & 120.5 & 120.5 \\
\hline 8 & 133.7 & 133.7 \\
\hline 9 & 181.1 & 181.1 \\
\hline 10 & 186.1 & 186.1 \\
\hline 11 & 196.6 & 196.6 \\
\hline 12 & 218.6 & 218.6 \\
\hline 13 & 228.3 & 228.3 \\
\hline 14 & 237.2 & 237.2 \\
\hline 15 & 245.5 & 245.5 \\
\hline 16 & 251.7 & 251.7 \\
\hline 17 & 265.5 & 265.5 \\
\hline 18 & 346.8 & 346.8 \\
\hline 19 & 363.7 & 363.7 \\
\hline 20 & 497.4 & 497.4 \\
\hline 21 & 538.8 & 538.8 \\
\hline 22 & 542.2 & 542.2 \\
\hline 23 & 550.2 & 550.2 \\
\hline 24 & 553.5 & 553.5 \\
\hline 25 & 558.2 & 558.2 \\
\hline 26 & 653.8 & 653.8 \\
\hline 27 & 659.2 & 659.2 \\
\hline 28 & 764.9 & 764.9 \\
\hline 29 & 776.3 & 776.3 \\
\hline 30 & 781.4 & 781.4 \\
\hline 31 & 841.0 & 841.0 \\
\hline 32 & 866.7 & 866.7 \\
\hline 33 & 868.2 & 868.2 \\
\hline 34 & 878.8 & 878.8 \\
\hline 35 & 891.0 & 891.0 \\
\hline 36 & 1221.1 & 1221.1 \\
\hline 37 & 1229.3 & 1229.3 \\
\hline 38 & 1240.7 & 1240.7 \\
\hline 39 & 1261.1 & 1261.1 \\
\hline 40 & 1280.1 & 1280.1 \\
\hline 41 & 1291.7 & 1291.7 \\
\hline 42 & 1431.3 & 1431.3 \\
\hline 43 & 1435.2 & 1435.2 \\
\hline 44 & 1447.2 & 1447.2 \\
\hline 45 & 1451.1 & 1451.1 \\
\hline 46 & 1454.1 & 1454.1 \\
\hline 47 & 1457.2 & 1457.2 \\
\hline 48 & 1462.1 & 1462.1 \\
\hline 49 & 1472.1 & 1472.1 \\
\hline 50 & 2199.8 & 2199.8 \\
\hline 51 & 2220.1 & 2220.1 \\
\hline 52 & 3086.7 & 3086.7 \\
\hline 53 & 3087.0 & 3087.0 \\
\hline 54 & 3088.9 & 3088.9 \\
\hline 55 & 3091.4 & 3091.4 \\
\hline 56 & 3210.2 & 3210.2 \\
\hline 57 & 3211.1 & 3211.1 \\
\hline 58 & 3223.2 & 3223.2 \\
\hline 59 & 3224.1 & 3224.1 \\
\hline 60 & 3224.8 & 3224.8 \\
\hline 61 & 3227.5 & 3227.5 \\
\hline 62 & 3235.5 & 3235.5 \\
\hline 63 & 3246.1 & 3246.1 \\
\hline
\end{tabular}




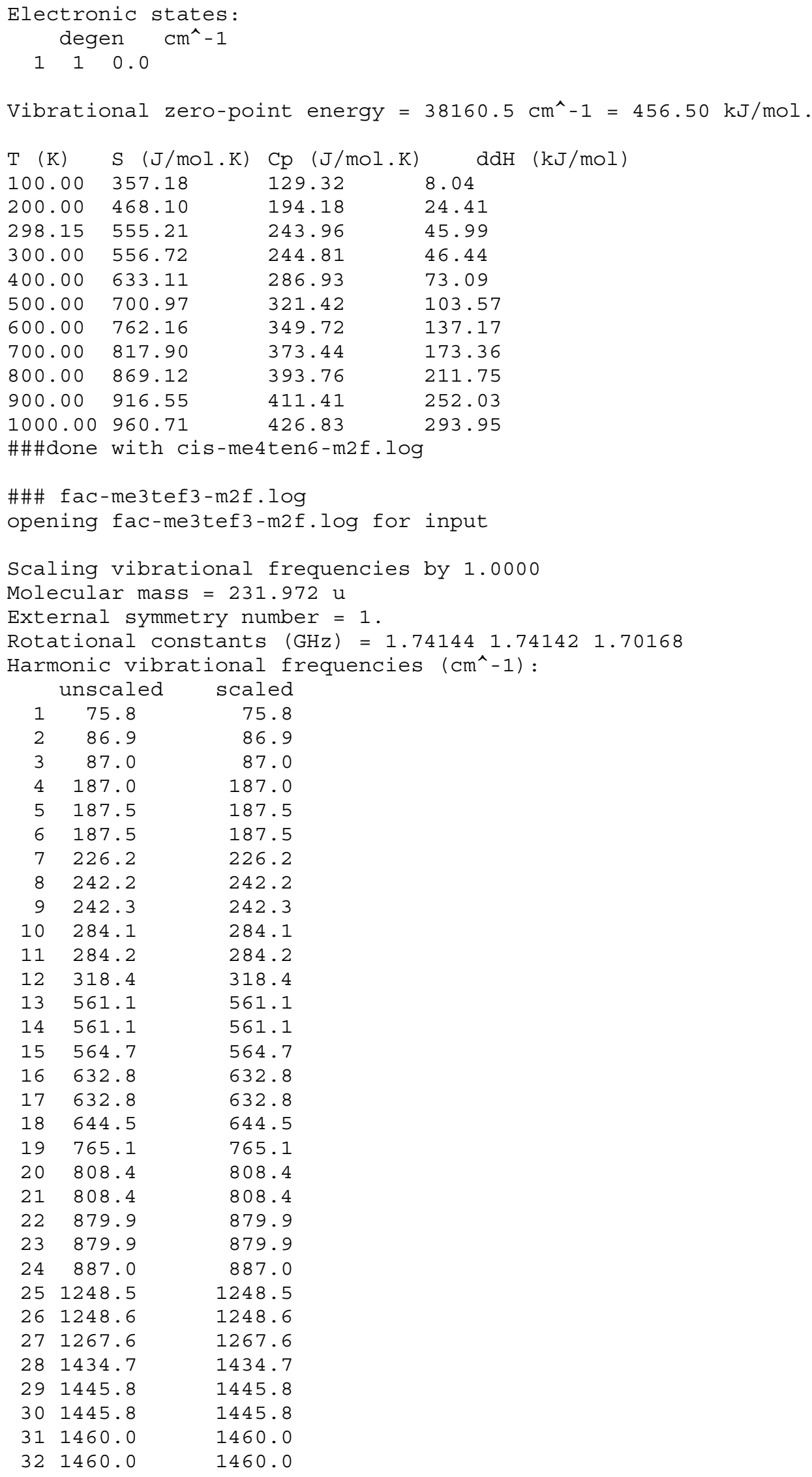




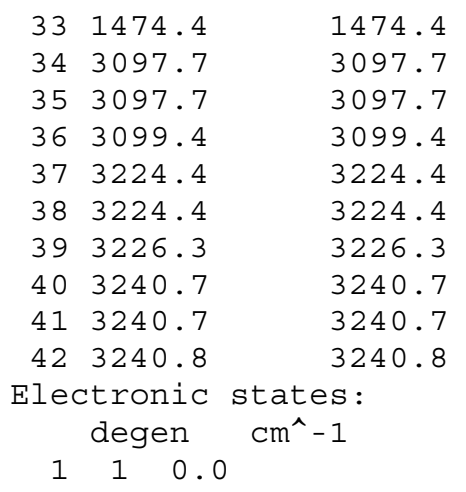




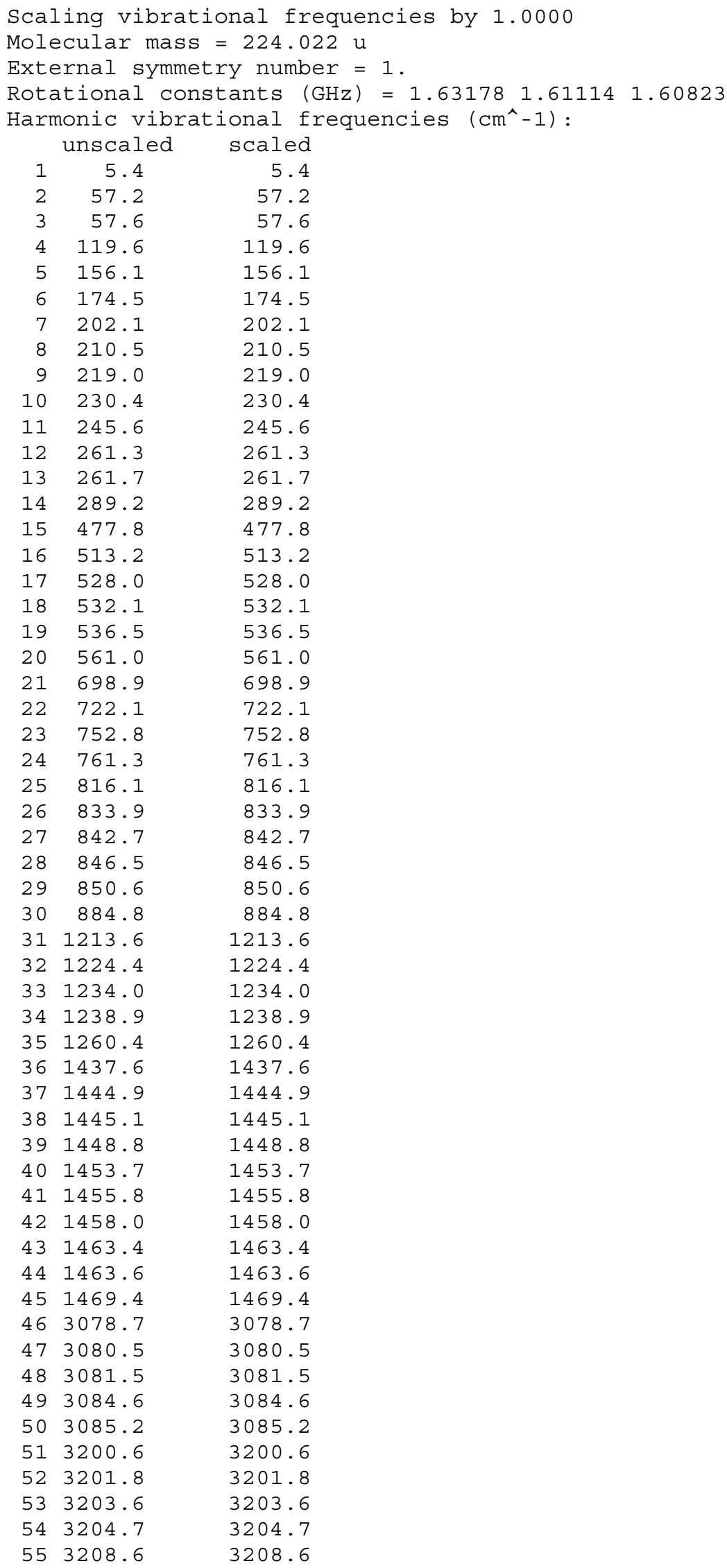




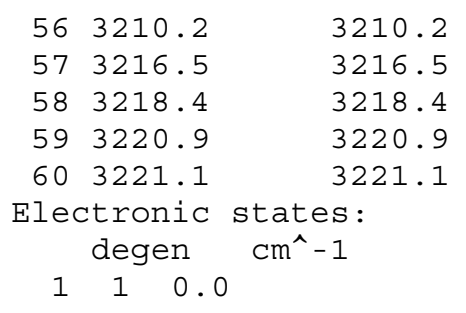




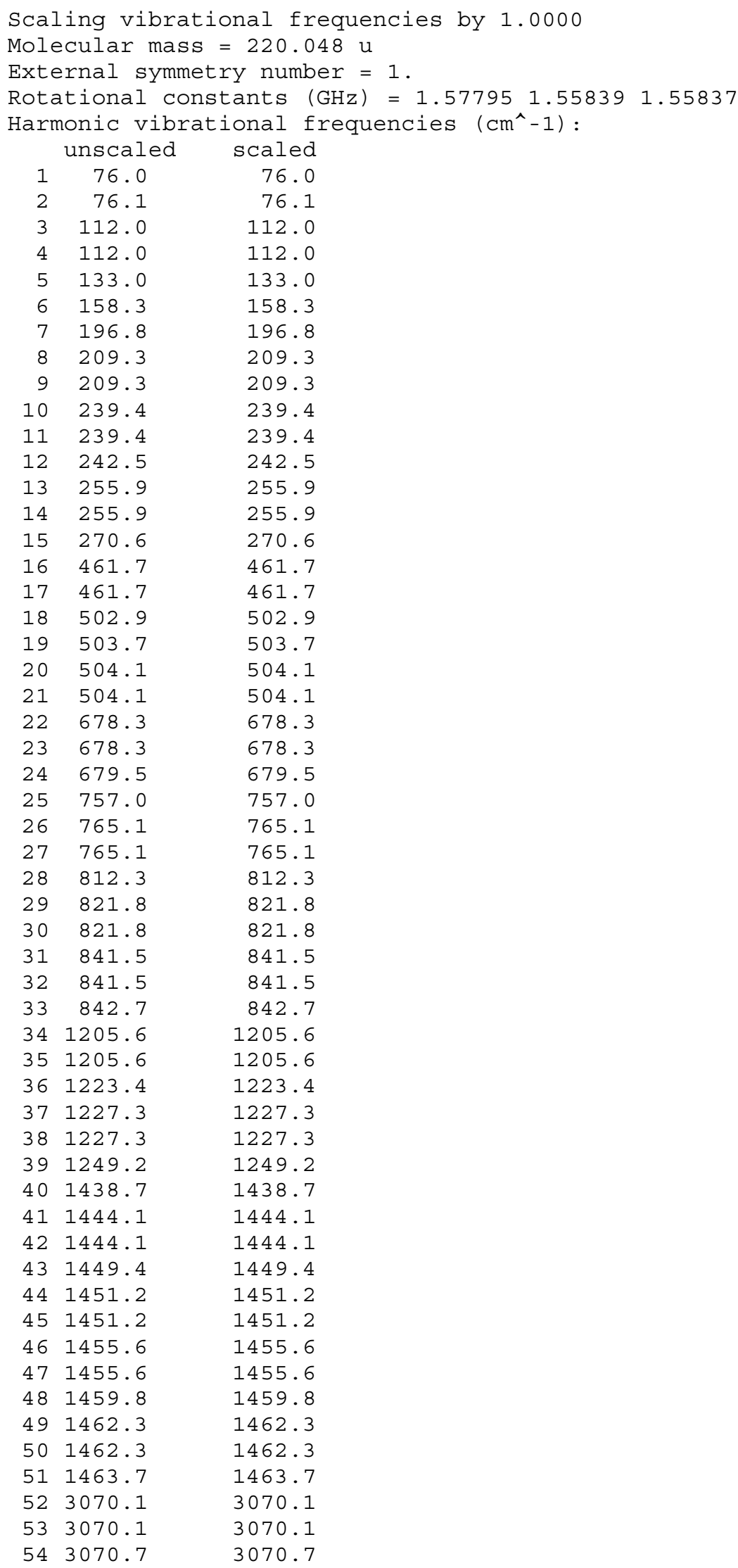




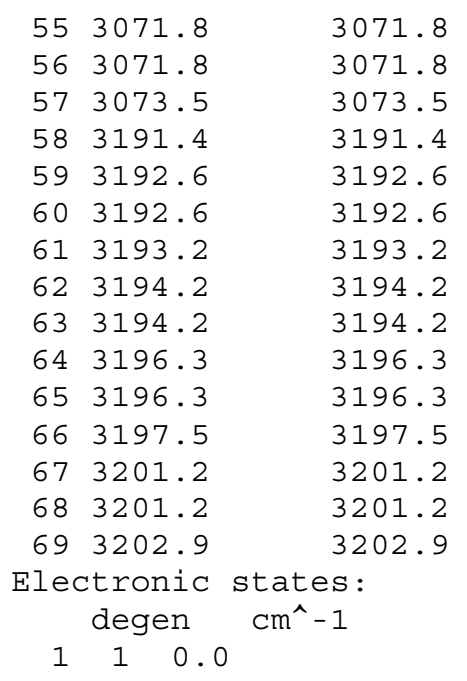




$\begin{array}{rrr}18 & 643.8 & 643.8 \\ 19 & 792.7 & 792.7 \\ 20 & 802.7 & 802.7 \\ 21 & 851.0 & 851.0 \\ 22 & 884.7 & 884.7 \\ 23 & 892.2 & 892.2 \\ 24 & 901.8 & 901.8 \\ 25 & 1251.8 & 1251.8 \\ 26 & 1257.6 & 1257.6 \\ 27 & 1266.4 & 1266.4 \\ 28 & 1444.3 & 1444.3 \\ 29 & 1444.5 & 1444.5 \\ 30 & 1452.3 & 1452.3 \\ 31 & 1454.7 & 1454.7 \\ 32 & 1459.0 & 1459.0 \\ 33 & 1460.1 & 1460.1 \\ 34 & 3095.5 & 3095.5 \\ 35 & 3109.2 & 3109.2 \\ 36 & 3109.3 & 3109.3 \\ 37 & 3224.1 & 3224.1 \\ 38 & 3227.2 & 3227.2 \\ 39 & 3240.4 & 3240.4 \\ 40 & 3240.4 & 3240.4 \\ 41 & 3258.7 & 3258.7 \\ 42 & 3258.7 & 3258.7 \\ \text { E1ectronic states } & \\ & \text { degen } & \text { cm } \\ 1 & 1 & 0.1 \\ & & \end{array}$

Vibrational zero-point energy $=26136.3 \mathrm{~cm}^{\wedge}-1=312.66 \mathrm{~kJ} / \mathrm{mol}$.

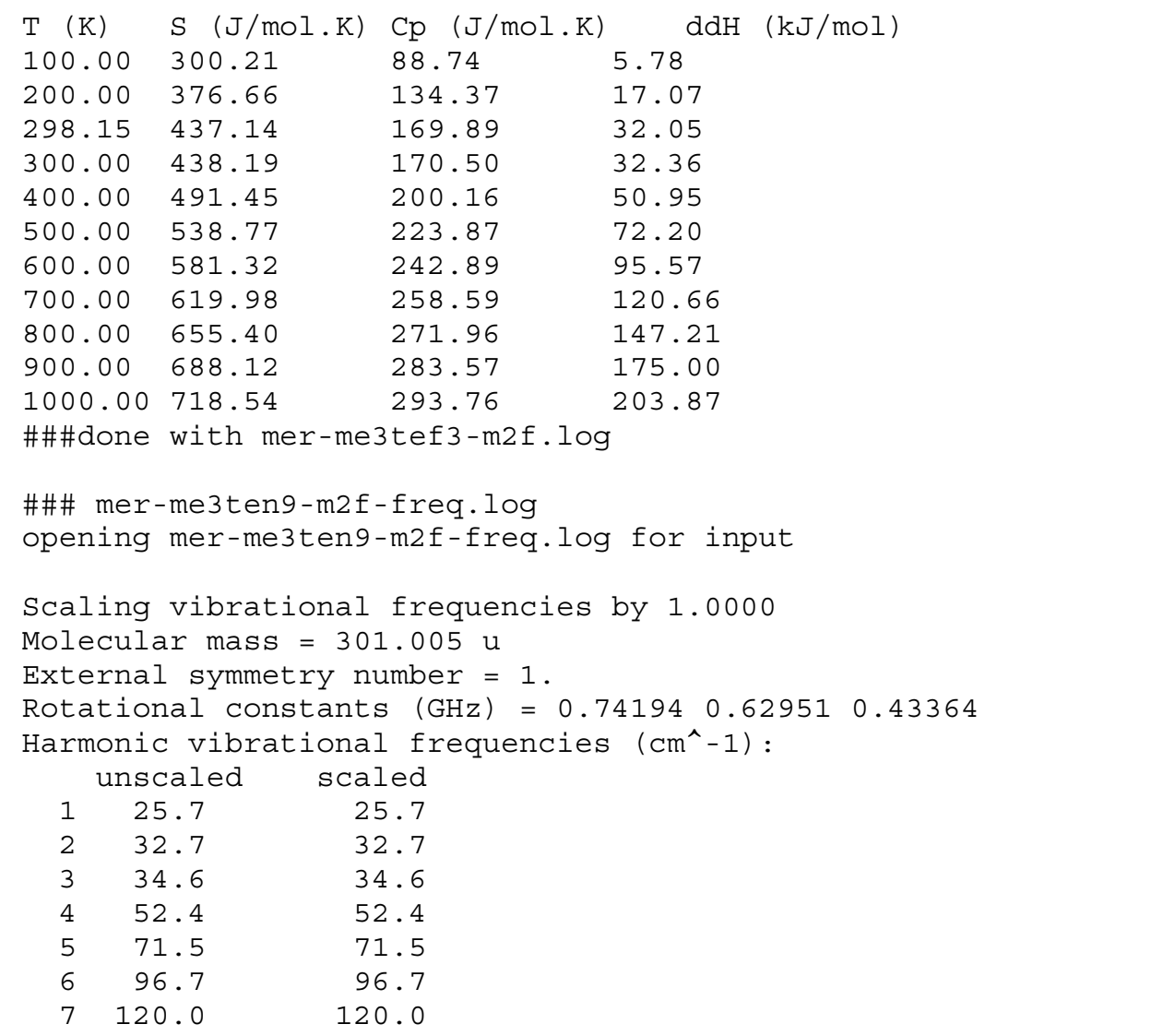




\begin{tabular}{|c|c|c|}
\hline 8 & 125.2 & 125.2 \\
\hline 9 & 133.0 & 133.0 \\
\hline 10 & 179.6 & 179.6 \\
\hline 11 & 183.1 & 183.1 \\
\hline 12 & 198.2 & 198.2 \\
\hline 13 & 214.1 & 214.1 \\
\hline 14 & 227.5 & 227.5 \\
\hline 15 & 242.4 & 242.4 \\
\hline 16 & 244.7 & 244.7 \\
\hline 17 & 253.7 & 253.7 \\
\hline 18 & 268.1 & 268.1 \\
\hline 19 & 356.0 & 356.0 \\
\hline 20 & 391.9 & 391.9 \\
\hline 21 & 412.5 & 412.5 \\
\hline 22 & 499.4 & 499.4 \\
\hline 23 & 548.2 & 548.2 \\
\hline 24 & 550.4 & 550.4 \\
\hline 25 & 552.6 & 552.6 \\
\hline 26 & 553.1 & 553.1 \\
\hline 27 & 559.8 & 559.8 \\
\hline 28 & 655.8 & 655.8 \\
\hline 29 & 661.2 & 661.2 \\
\hline 30 & 663.8 & 663.8 \\
\hline 31 & 788.3 & 788.3 \\
\hline 32 & 820.0 & 820.0 \\
\hline 33 & 832.3 & 832.3 \\
\hline 34 & 867.1 & 867.1 \\
\hline 35 & 873.3 & 873.3 \\
\hline 36 & 906.5 & 906.5 \\
\hline 37 & 1219.3 & 1219.3 \\
\hline 38 & 1221.2 & 1221.2 \\
\hline 39 & 1257.1 & 1257.1 \\
\hline 40 & 1279.0 & 1279.0 \\
\hline 41 & 1281.6 & 1281.6 \\
\hline 42 & 1287.1 & 1287.1 \\
\hline 43 & 1432.9 & 1432.9 \\
\hline 44 & 1440.9 & 1440.9 \\
\hline 45 & 1446.9 & 1446.9 \\
\hline 46 & 1450.5 & 1450.5 \\
\hline 47 & 1456.9 & 1456.9 \\
\hline 48 & 1460.7 & 1460.7 \\
\hline 49 & 2184.9 & 2184.9 \\
\hline 50 & 2217.1 & 2217.1 \\
\hline 51 & 2231.7 & 2231.7 \\
\hline 52 & 3093.8 & 3093.8 \\
\hline 53 & 3094.0 & 3094.0 \\
\hline 54 & 3095.7 & 3095.7 \\
\hline 55 & 3226.9 & 3226.9 \\
\hline 56 & 3230.6 & 3230.6 \\
\hline 57 & 3234.8 & 3234.8 \\
\hline 58 & 3236.4 & 3236.4 \\
\hline 59 & 3238.9 & 3238.9 \\
\hline 60 & 3251.3 & 3251.3 \\
\hline & \multicolumn{2}{|c|}{$\begin{array}{l}\text { ctronic states: } \\
\text { degen } \mathrm{cm}^{\wedge}-1\end{array}$} \\
\hline 1 & 10.0 & \\
\hline
\end{tabular}

Vibrational zero-point energy $=32883.0 \mathrm{~cm}^{\wedge}-1=393.37 \mathrm{~kJ} / \mathrm{mol}$.

$\mathrm{T}(\mathrm{K}) \quad \mathrm{S}(\mathrm{J} / \mathrm{mol} . \mathrm{K}) \quad \mathrm{Cp}(\mathrm{J} / \mathrm{mol} . \mathrm{K}) \quad \mathrm{ddH}(\mathrm{kJ} / \mathrm{mol})$

$\begin{array}{llll}100.00 & 375.53 & 138.05 & 8.73\end{array}$ 


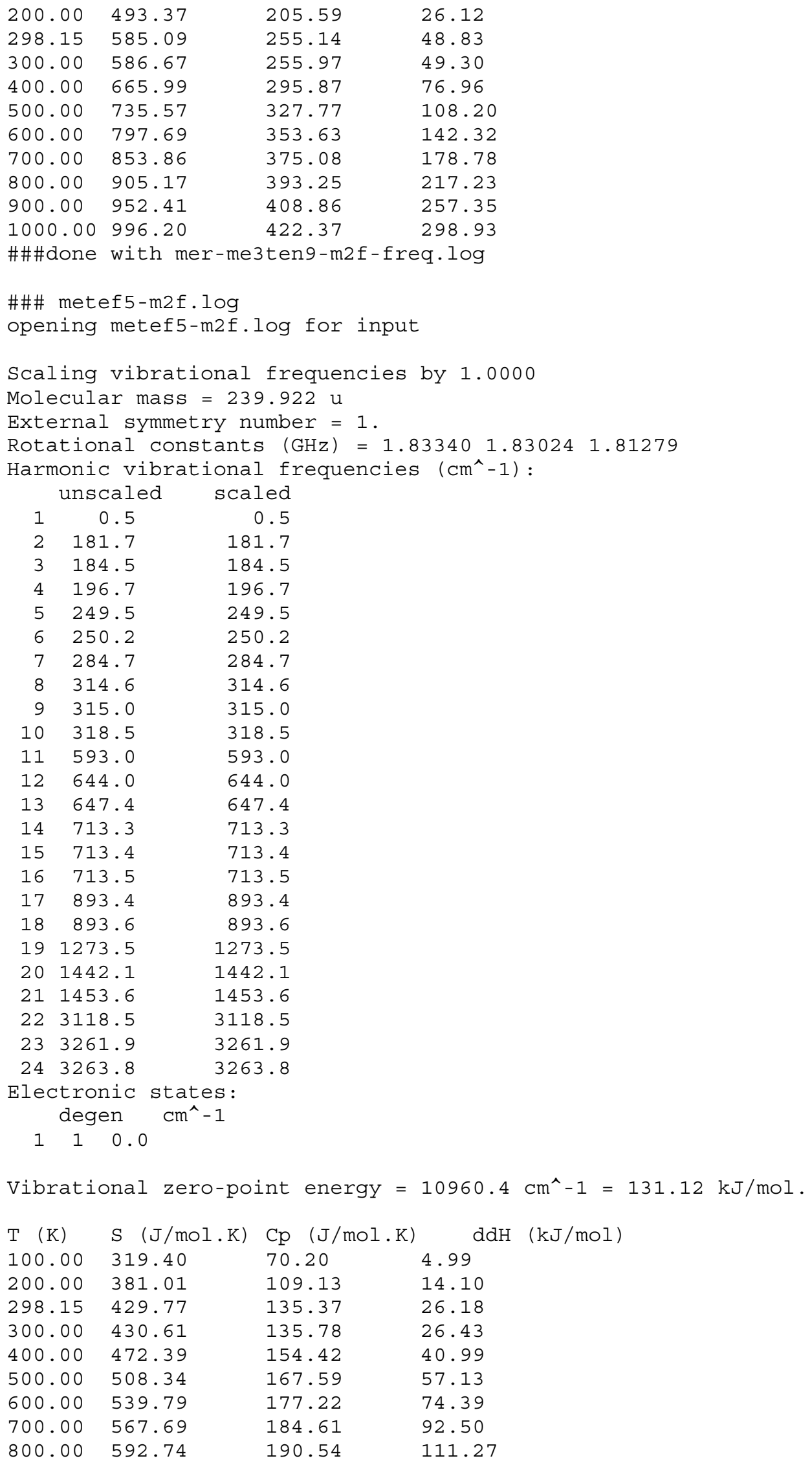




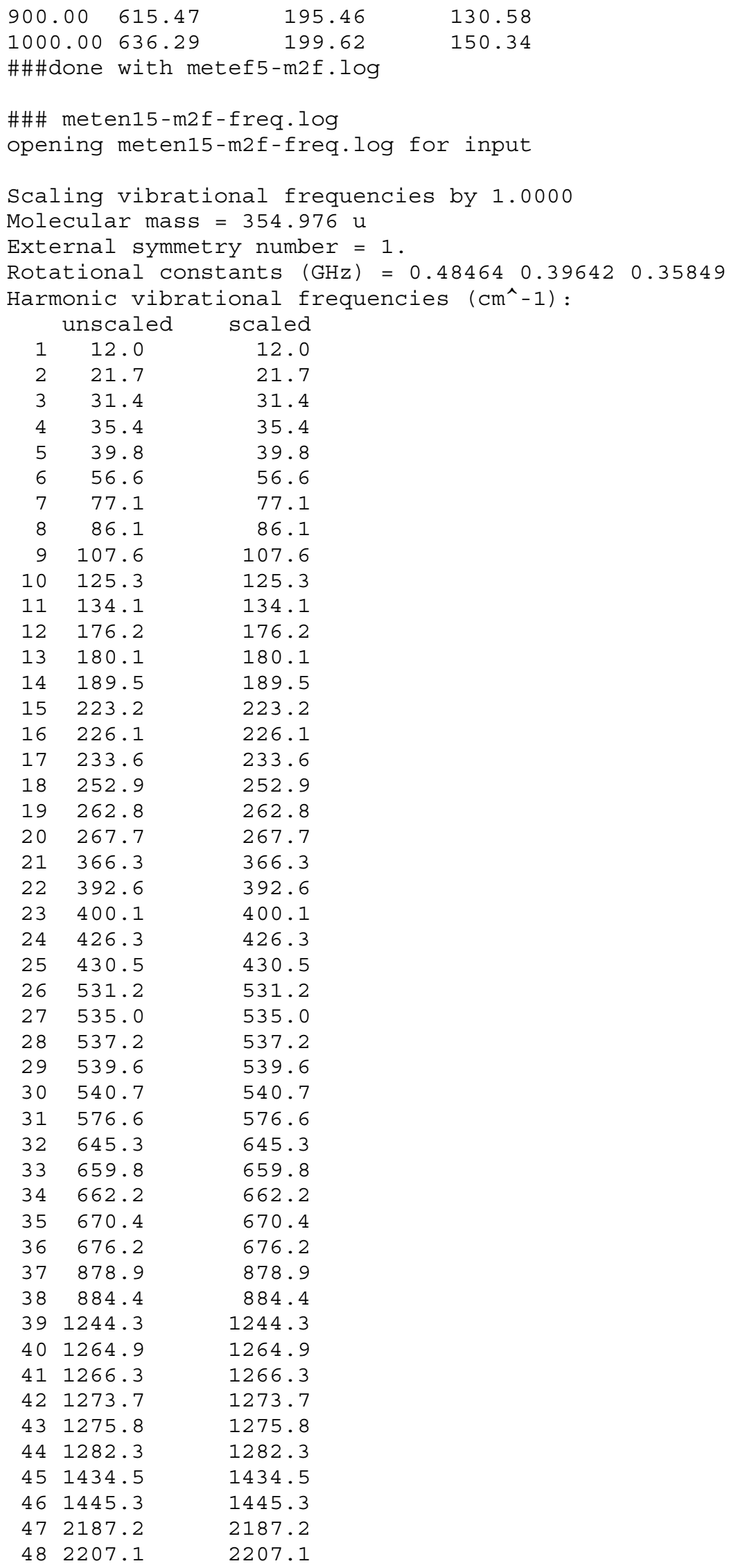




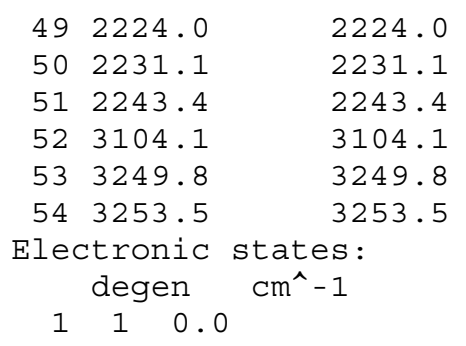




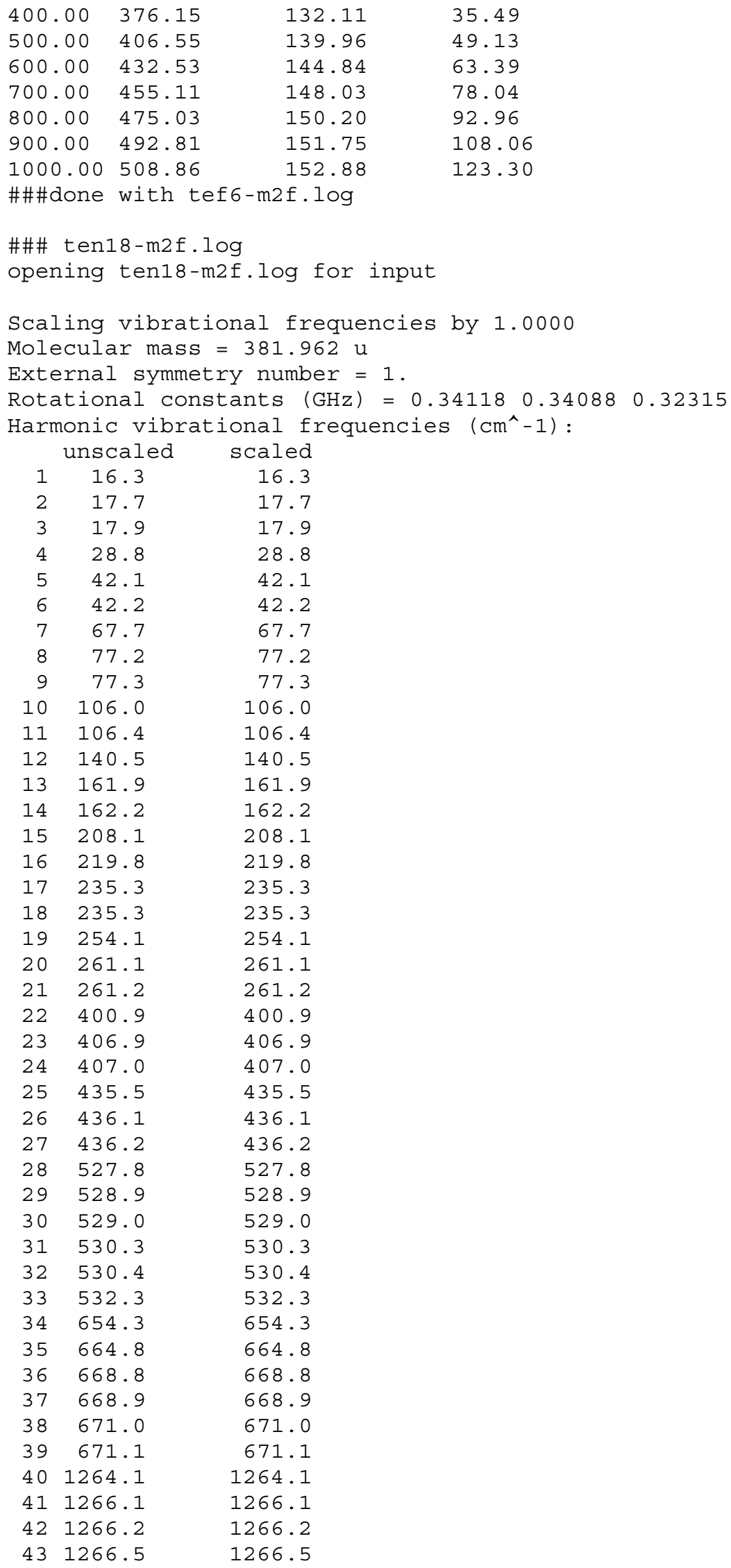




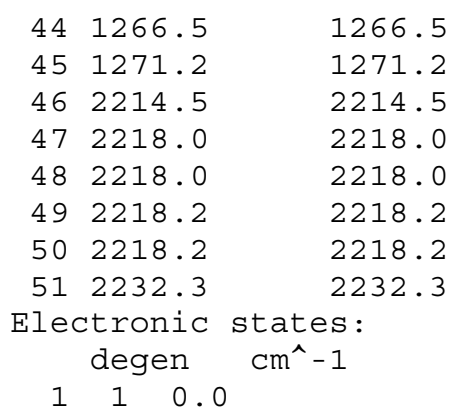




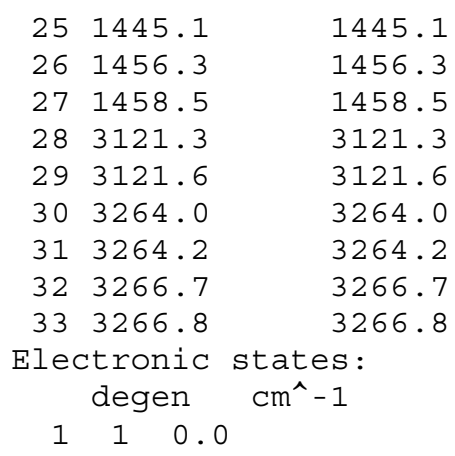




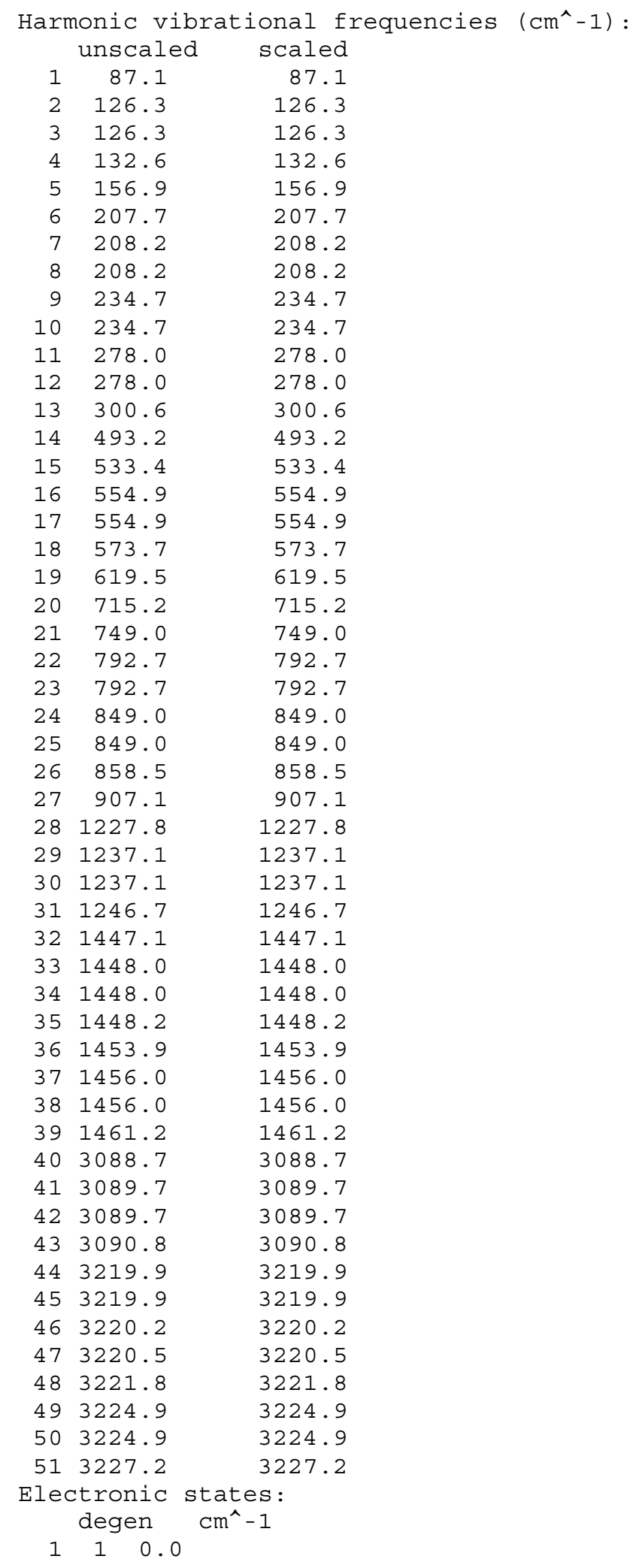

Vibrational zero-point energy $=33563.6 \mathrm{~cm}^{\wedge}-1=401.51 \mathrm{~kJ} / \mathrm{mol}$.

$\mathrm{T}(\mathrm{K}) \quad \mathrm{S}(\mathrm{J} / \mathrm{mol} . \mathrm{K}) \quad \mathrm{Cp}(\mathrm{J} / \mathrm{mol} . \mathrm{K}) \quad \mathrm{ddH} \quad(\mathrm{kJ} / \mathrm{mol})$

$\begin{array}{llll}100.00 & 293.16 & 92.81 & 5.61\end{array}$ 


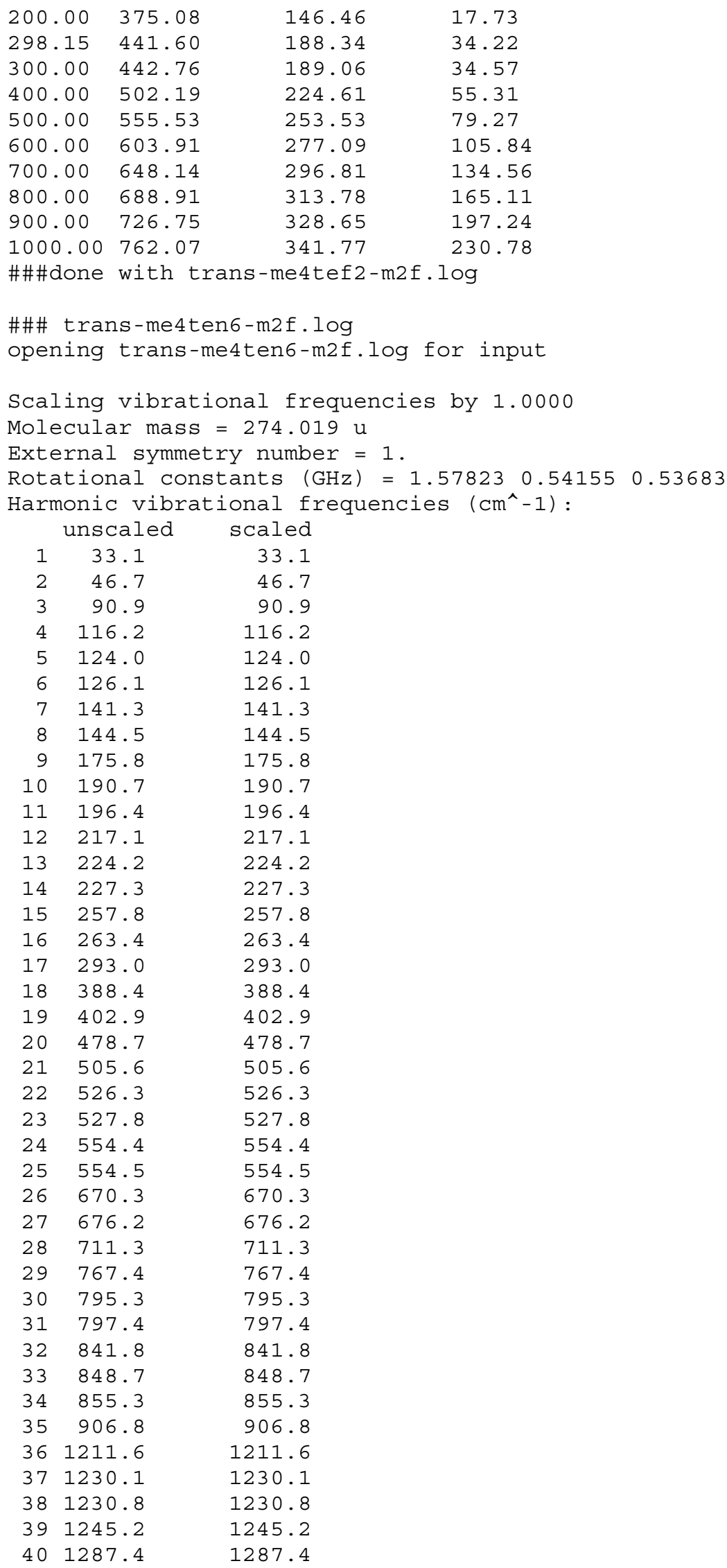


